\title{
Covariant density functional theory input for r-process simulations in actinides and superheavy nuclei: the ground state and fission properties
}

\author{
A. Taninah, ${ }^{1}$ S. E. Agbemava, ${ }^{1,2}$ and A. V. Afanasjev ${ }^{1}$ \\ ${ }^{1}$ Department of Physics and Astronomy, Mississippi State University, MS 39762 \\ ${ }^{2}$ Ghana Atomic Energy Commission, National Nuclear Research Institute, P.O.Box LG80, Legon, Ghana.
}

(Dated: November 30, 2020)

\begin{abstract}
The systematic investigation of the ground state and fission properties of even-even actinides and superheavy nuclei with $Z=90-120$ from the two-proton up to two-neutron drip lines with proper assessment of systematic theoretical uncertainties has been performed for the first time in the framework of covariant density functional theory (CDFT). These results provide a necessary theoretical input for the r-process modeling in heavy nuclei and, in particular, for the study of fission cycling. Four state-of-the-art globally tested covariant energy density functionals (CEDFs), namely, DD-PC1, DD-ME2, NL3* and PC-PK1, representing the major classes of the CDFT models are employed in the present study. Ground state deformations, binding energies, two neutron separation energies, $\alpha$-decay $Q_{\alpha}$ values and half-lives and the heights of fission barriers have been calculated for all these nuclei. Theoretical uncertainties in these physical observables and their evolution as a function of proton and neutron numbers have been quantified and their major sources have been identified. Spherical shell closures at $Z=120, N=184$ and $N=258$ and the structure of the single-particle (especially, high- $j$ ) states in their vicinities as well as nuclear matter properties of employed CEDFs are two major factors contributing into theoretical uncertainties. However, different physical observables are affected in a different way by these two factors. For example, theoretical uncertainties in calculated ground state deformations are affected mostly by former factor, while theoretical uncertainties in fission barriers depend on both of these factors.
\end{abstract}

\section{INTRODUCTION}

The majority of the nuclei found in nature are formed in the astrophysical rapid neutron-capture process ( $\mathrm{r}-$ process). Indeed, the r-process is responsible for the synthesis of approximately half of the nuclei in nature beyond $\mathrm{Fe}[1]$ and it is the only process which leads to the creation of nuclei heavier than $\mathrm{Bi}[2]$. It takes place at extremely high neutron densities $\left(N_{n} \geq 10^{20} \mathrm{~cm}^{-3}\right)$ which are high enough to make neutron capture faster than $\beta$ decay even for the nuclei with neutron excess between 15 to 30 units from the stability line. The production of neutron-rich nuclei located in the vicinity of the neutron dripline is enabled under these conditions via neutron capture and $(\gamma, n)$ photodisintegration during the r-process. Once the neutron source ceases, the progenitor nuclei decay either via $\beta^{-}$decay or $\alpha$ emission or by fission processes (such as neutron-induced, $\beta$-delayed and spontaneous fissions) towards stability and form the stable isotopes of elements up to the heaviest species Th, $\mathrm{U}$ and $\mathrm{Pu}$. The typical timescale of the r-process is in the seconds range [2-4].

Over the years different possible astrophysical sites have been and still are considered as possible candidates for the r-process. These include core-collapse supernovae, magneto-rotational core-collapse supernovae, accretion disk outflows from collapsars, neutron star (NS) mergers and neutron star - black hole mergers etc [2-5]. So far only the NS merger is experimentally confirmed as a site of the r-process via the observation of gravitational waves from the GW170817 neutron star merger [6] with simultaneous observation of the AT 2017gfo macronova/kilonova afterglow [7]. In NS mergers, the r-process material originates in the NS crust, and the composition of the crust and how it responds to stress caused by the merger dictates the amount of the r-process material which is ejected. NS merger produces approximately $10^{-2} \mathrm{M}_{\odot}$ of ejected r-process matter in the dynamic ejecta and similar amount in the accretion disk outflows [4, 8, 9]. Although some uncertainties still exist, at present the NS merger is considered as the major astrophysical site of the r-process providing the dominant source of heavy nuclei $[2,4,5]$.

The modeling of the r-process in such neutron-rich environments depends sensitively on nuclear masses, $\alpha$ - and $\beta$-decay half-lives, neutron capture and fission properties of the nuclei the majority of which will never be measured in laboratory conditions $[2,4,5,10]$. Nuclear masses determine the flow path of the $\mathrm{r}$-process, $\beta$-decay rates are responsible for the speed with which the r-process moves matter to heavier nuclei, $\alpha$-decays become important in heavy nuclei as a competing decay channel and neutron captures drive the nuclei towards neutron-rich side of nuclear landscape. Of special interest in the context of the present manuscript are fission properties. Fission needs to be considered in the r-process simulations if the neutron-to-seed ratio is large enough to produce fissioning nuclei [11-14]. If the initial neutron-to-seed ratio is large $(\geq 100)$ the $r$-process can reach the region near and beyond neutron shell closure at $N=184$, where fission plays a dominant role (the examples of the distribution of abundances of actinides and superheavy elements as obtained in a pair of the r-process simulations are shown in Fig. 1). This is exactly the case for the NS mergers [12]. In this case, all fission channels (neutron induced, betadelayed, neutrino induced and spontaneous fissions) need 
to be considered. Fission leads to the termination of hot r-process by means of fission-cycling which returns matter to lighter nuclei [10]. It also determines the strength of fission cycling, the ratio of the actinides to light and medium mass r-process nuclei and thus the shape of final element abundance pattern. In addition, it defines the possibility of the formation of neutron-rich superheavy nuclei in the r-process [15].

Thus, in the situation when experimental data are not known the outcome of the r-process modeling sensitively depends on the quality of employed theoretical frameworks and associated theoretical uncertainties and their propagation on going to neutron-rich nuclei. By tradition, the output of different theoretical frameworks is used for different physical observables (such as masses, the rates and half-lives of different decay channels and reactions etc.) in the the r-process modeling. Existing r-process calculations, which include information on fission properties, are based on the fission barrier heights obtained in non-relativistic models [10, 14, 18]. So far fission barrier heights obtained in finite range droplet model (FRDM), Thomas-Fermi (TF) model and extended Thomas-Fermi model with Strutinsky integral approach (ETFSI-Q), Hartree-Fock-Bogoliubov (HFB) model with Skyrme HFB-14 energy density functional (EDF) and BCPM EDF have been used in these calculations. Moreover the sets of fission barriers relevant for the r-process simulations have been generated in the FRDM in Ref. [18], in the ETFSI-Q approach with the SkSC4 functional in Ref. [19], in the HFB models with Skyrme HFB-14, SV-min, SLy6, SkI3, SV-bas EDFs [2022], Gogny D1M* [23] and BCPM [18] functionals. Note that all these calculations assume axial symmetry of the nuclei.

Covariant density functional theory (CDFT) [24] is an approach alternative to above mentioned non-relativistic methods and so far it has not been applied for a systematic study of fission properties of the nuclei relevant for the r-process modeling. However, this theory has a number of advantages over non-relativistic methods which are discussed below. Covariant energy density functionals (CEDF) exploit basic properties of QCD at low energies, in particular symmetries and the separation of scales [25]. They provide a consistent treatment of the spin degrees of freedom and spin-orbit splittings ([26, 27]); the latter has an essential influence on the underlying shell structure. In addition, these functionals include nuclear magnetism [28], i.e. a consistent description of currents and time-odd mean fields important for odd-mass nuclei [29], the excitations with unsaturated spins, magnetic moments [30] and nuclear rotations [31, 32]. Because of Lorentz invariance no new adjustable parameters are required for the time-odd parts of the mean fields [29]. This is contrary to the case of non-relativistic Skyrme DFTs in which several prescriptions for fixing time-odd mean fields exist
$[33,34]^{1}$. This fact could be extremely important in the applications to fission processes including dynamical correlations since time-odd mean fields have a significant impact on collective masses $[35,36]$.

The goal of this study is to close this gap in our knowledge and to perform first systematic investigation within the CDFT framework of the ground state and fission properties of the nuclei with proton numbers $Z=90-120$ located between two-proton and two-neutron drip lines (see Fig. 1). This study will not only provide an input for the r-process modeling but also evaluate the extension of nuclear landscape up to two-neutron drip line as well as estimate relevant theoretical uncertainties and their sources in the description of physical observables of interest. In addition, it will allow for the first time to compare the predictions for fission barriers in the nuclei relevant for r-process modeling obtained in relativistic and non-relativistic frameworks.

Considering the region of the nuclear chart in which the r-process is expected to take place and the fact that there are no experimental data to benchmark theoretical results, it is important to estimate theoretical uncertainties in the predictions of physical observables of interest [37-39]. Theoretical uncertainties emerge from the underlying theoretical approximations. In the DFT framework, there are two major sources of these approximations, namely, the range of interaction and the form of the density dependence of the effective interaction $[40,41]$. In the non-relativistic case one has zero range Skyrme and finite range Gogny forces and different density dependencies [40]. A similar situation exists also in the relativistic case: point coupling and meson exchange models have an interaction of zero and of finite range, respectively [24, 42-44]. The density dependence is introduced either through an explicit dependence of the coupling constants $[42,44,45]$ or via non-linear meson couplings $[41,43]$. This ambiguity in the definition of the range of the interaction and its density dependence leads to several major classes of the covariant energy density functionals (CEDF) which were discussed in Ref. [39].

Since statistical uncertainties in the physical observables are smaller than systematic ones (see Ref. [46]), we focus here on the latter ones. They are related to the choice of EDF. We follow our previous publications on this topic [39, 47-49] and define systematic theoretical uncertainty for a given physical observable (which we call in the following "spreads") via the spread of theoretical predictions as [39]

$$
\Delta O(Z, N)=\left|O_{\max }(Z, N)-O_{\min }(Z, N)\right|,
$$

where $O_{\max }(Z, N)$ and $O_{\min }(Z, N)$ are the largest and smallest values of the physical observable $O(Z, N)$ obtained within the set of CEDFs under investigation for

\footnotetext{
1 Unfortunately, the role of time-odd mean fields in Gogny DFTs has not been studied so far and it is unknown whether they are uniquely defined.
} 


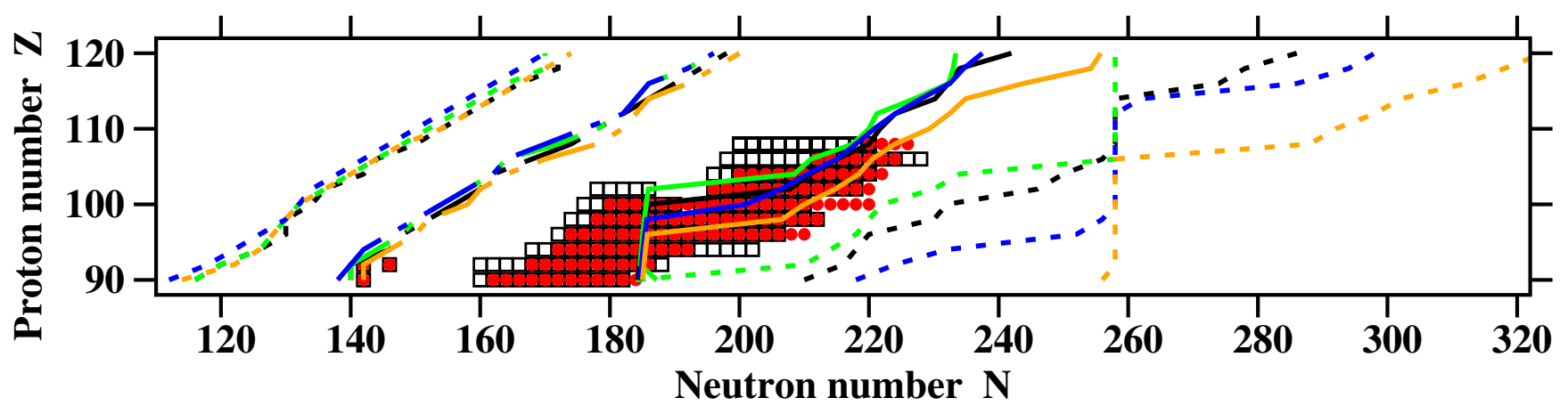

FIG. 1. The part of nuclear chart under study. Black, green, blue and orange lines are used for the results obtained with the DD-PC1, DD-ME2, NL3* and PC-PK1 CEDFs, respectively. Two-proton and two-neutron drip lines predicted by four CEDFs are shown by dashed lines. Two samples of the distribution of the abundances of heavy and superheavy elements in the r-process simulations are shown by open squares (based on bottom panel of Fig. 8 of Ref. [15]) and red circles (based on Fig. 3 of Ref. [16]). The former results correspond to hot r-process conditions and are based on the ETFS/ETFSI combination of fission barriers and mass predictions (see Ref. [15]). The latter results have been obtained in Ref. [16] based on fission properties obtained with the BCPM energy density functional. Note that these r-process calculations are restricted to the $Z \leq 110$ nuclei. The r-process path is shown here approximately by solid lines corresponding to two-neutron separation energy $S_{2 n}=4.0 \mathrm{MeV}^{\mathrm{a}}$. Dot-dashed lines show the beta-stability lines for four functionals.

a The r-process proceeds along the lines of constant neutron separation energies towards heavy nuclei that for typical conditions during the r-process corresponds to $S_{n}^{0}$ approximately located between 2 and $3 \mathrm{MeV}[1,17]$. However, due to neutron pairing being stronger at even neutron numbers $N$ the most abundant isotopes always have even $N$ values. For this reason, we follow Refs. [1, 17] and characterize the r-process path [the path in the $(Z, N)$ plane corresponding to an isotope with highest abundance in each isotopic chain] as the path which satisfies the condition that two-neutron separation energy $S_{2 n}$ has the value $S_{2 n}=2 S_{n}^{0}$.

the $(Z, N)$ nucleus. Note that these spreads are only a crude approximation to the systematic theoretical errors discussed in Ref. [38] since they are obtained with a very small number of functionals which do not form an independent statistical ensemble. Note also that these systematic errors are not well defined in unknown regions of nuclear chart or deformation since systematic biases of theoretical models could not be established in these regions in the absence of experimental data and/or an exact theory.

In order to consider several possible scenarios in the evolution of physical observables as a function of proton and neutron numbers and to evaluate systematic theoretical uncertainties, the CEDFs NL3* [43], DD-ME2 [42], DD-PC1 [44] and PC-PK1 [50] are used here ${ }^{2}$ for all $Z=90-120$ even-even nuclei located between two-

\footnotetext{
2 The compilation of Ref. [51] published in 2014 indicates the existence of 263 CEDFs ranging from simplest ansatz non-linear meson exchange functionals such as NL1 [52] and NL3* [43] to more microscopically motivated CEDFs such as G1, G2 [53] and $\mathrm{DD}-\mathrm{ME} \delta$ [54]. In addition, a number of new functionals were fitted in the time period between 2014 and 2020 (see, for example, Refs. $[46,55-58])$ so at present the total number of available CEDFs is likely to be in the vicinity of 300 . Because of extremely time-consuming nature of numerical calculations in this project, we use only the indicated last generation functionals. They outperform previous generation functionals in terms of the accuracy of global description of ground state observables such as binding energies and charge radii $[39,49,59,60]$, properly describe the regions of octupole deformation $[48,61]$ and are able to reproduce experimentally known fission barriers in actinides [62-64].
}

proton and two-neutron drip lines ${ }^{3}$. These are stateof-the-art functionals representing the major classes of CDFTs (for more details see the discussion in Sect. II of Ref. [39] and the introduction to Ref. [59]). Their performance and related theoretical uncertainties have recently been analyzed globally in Refs. [39, 48, 49, 68] and in particular in superheavy nuclei in Refs. [47, 59]. They are characterized by an improved accuracy of the description of experimental data as compared with the previous generation of CEDFs. The fact that the NL3*, DD-PC1 and PC-PK1 functionals reproduce empirical data on fission barrier heights in actinides [62-64, 69] is especially important in the context of the present study.

The manuscript is organized as follows. Theoretical framework and the details related to the calculations of the ground states and fission barriers are discussed in Sec. II. Sec. III is devoted to the analysis of the results

\footnotetext{
${ }^{3}$ Present study partially builds on previous results obtained by us. These are ground state properties of the $Z=90-104$ nuclei located between two-proton and two-neutron drip lines obtained in reflection symmetric RHB calculations with the NL3*, DDME2 and DD-PC1 CEDFs in Ref. [39], ground state properties of octupole deformed nuclei with $N<210$ obtained in reflection asymmetric RHB calculations with NL3*, DD-ME2, DD-PC1 and PC-PK1 functionals in Ref. [48, 61] and ground state properties and the heights of inner fission barriers of superheavy nuclei with $Z=100-120, N \leq 196$ obtained in reflection symmetric RHB calculations with NL3*, DD-ME2, DD-PC1 and PC-PK1 functionals in Refs. $[47,59]$. Additional information on the extension of nuclear landscape to $Z>120$ obtained with DD-PC1 CEDF can be found in Refs. [65-67].
} 
of the calculations for ground state properties. Theoretical results for $\alpha$-decay properties and related theoretical uncertainties are presented in Sec. IV. The heights of primary fission barriers, their distribution in the $(Z, N)$ plane, related theoretical uncertainties and the comparison with non-relativistic results are considered in Sec. V. Finally, Sec. VI summarizes the results of our work.

\section{THEORETICAL FRAMEWORK}

In the present manuscript, the RHB framework with finite range pairing and its separable limit are used for a systematic study of ground state properties of all eveneven actinides $(Z=90-102)$ and superheavy $(Z=$ $104-120)$ nuclei from the proton to neutron drip line. It has the proper coupling to the continuum at the neutron drip line and, therefore, it allows a correct description of weakly bound nuclei close to the neutron drip line.

TABLE I. The nuclei in which extremely superdeformed minimum is the lowest in energy in the calculations with CEDF DD-PC1. The columns 1 and 2 show the proton and neutron numbers of the nuclei. Third column displays the energy $E_{N-S}$ [in $\mathrm{MeV}$ ] by which the ESD minimum is lower than the normal-deformed minimum. The deformations of the ESD minimum $\beta_{2}^{\text {min }}$ and the saddle of second fission barrier $\beta_{2}^{\text {saddle }}, \beta_{3}^{\text {saddle }}$ are presented in the columns 4 and 5 , respectively. The energies [in $\mathrm{MeV}$ ] of the second fission barrier with respect of the ESD minimum, obtained in the RSRHB and RA-RHB calculations, are shown in the columns 6 and 7 . Note that the values presented in the columns 5-7 are obtained in the calculations with $N_{F}=26$; this is done in order to have a comparable numerical accuracy with the one obtained at normal deformed minimum.

\begin{tabular}{|c|c|c|c|c|c|c|}
\hline $\mathrm{Z}$ & $\mathrm{N}$ & $\begin{array}{c}E_{N-S} \\
{[\mathrm{MeV}]}\end{array}$ & $\beta_{2}^{\text {min }}$ & $\beta_{2}^{\text {saddle }}, \beta_{3}^{\text {saddle }}$ & $\begin{array}{c}E_{B}^{I I}[\mathrm{MeV}] \\
(\mathrm{RS}-\mathrm{RHB})\end{array}$ & $\begin{array}{c}E_{B}^{I I}[\mathrm{MeV}] \\
(\mathrm{RA}-\mathrm{RHB})\end{array}$ \\
\hline 1 & 2 & 3 & 4 & 5 & 6 & 7 \\
\hline 98 & 142 & 0.603 & 0.88 & $1.21,0.33$ & 7.207 & 2.625 \\
\hline 98 & 144 & 0.306 & 0.90 & $1.34,0.46$ & 7.373 & 3.503 \\
\hline 98 & 228 & 2.341 & 1.01 & $1.29,0.34$ & 6.622 & 2.906 \\
\hline 98 & 230 & 2.083 & 1.01 & $1.30,0.35$ & 5.072 & 3.203 \\
\hline 100 & 146 & 0.876 & 0.97 & $1.32,0.37$ & 5.890 & 2.985 \\
\hline 100 & 230 & 2.431 & 1.01 & $1.30,0.35$ & 5.750 & 3.270 \\
\hline 100 & 232 & 2.336 & 1.01 & $1.30,0.37$ & 4.060 & 2.739 \\
\hline 102 & 146 & 2.038 & 0.99 & $1.32,0.34$ & 4.813 & 2.749 \\
\hline 102 & 148 & 0.629 & 0.98 & $1.28,0.31$ & 4.093 & 2.250 \\
\hline 102 & 232 & 2.591 & 1.02 & $1.29,0.36$ & 3.476 & 2.840 \\
\hline 102 & 234 & 3.567 & 1.03 & $1.31,0.31$ & 2.674 & 2.304 \\
\hline 104 & 146 & 3.435 & 0.99 & $1.33,0.28$ & 6.042 & 2.410 \\
\hline 104 & 148 & 1.921 & 1.00 & $1.31,0.27$ & 5.579 & 2.204 \\
\hline 104 & 150 & 0.924 & 1.00 & $1.29,0.27$ & 5.370 & 2.505 \\
\hline 106 & 148 & 3.638 & 1.08 & $1.32,0.26$ & 4.112 & 2.340 \\
\hline 106 & 150 & 2.374 & 1.10 & $1.30,0.23$ & 3.753 & 2.345 \\
\hline
\end{tabular}

The RHB equations for the fermions are given by [70]

$$
\left(\begin{array}{cc}
\hat{h}_{D}-\lambda & \hat{\Delta} \\
-\hat{\Delta}^{*} & -\hat{h}_{D}^{*}+\lambda
\end{array}\right)\left(\begin{array}{l}
U(\boldsymbol{r}) \\
V(\boldsymbol{r})
\end{array}\right)_{k}=E_{k}\left(\begin{array}{l}
U(\boldsymbol{r}) \\
V(\boldsymbol{r})
\end{array}\right)_{k},
$$

Here, $\hat{h}_{D}$ is the Dirac Hamiltonian for the nucleons with mass $m ; \lambda$ is the chemical potential defined by the constraints on the average particle number for protons and neutrons; $U_{k}(\boldsymbol{r})$ and $V_{k}(\boldsymbol{r})$ are quasiparticle Dirac spinors [70-72] and $E_{k}$ denotes the quasiparticle energies. The Dirac Hamiltonian

$$
\hat{h}_{D}=\boldsymbol{\alpha}(\boldsymbol{p}-\boldsymbol{V})+V_{0}+\beta(m+S) .
$$

contains an attractive scalar potential

$$
S(\boldsymbol{r})=g_{\sigma} \sigma(\boldsymbol{r}),
$$

a repulsive vector potential

$$
V_{0}(\boldsymbol{r})=g_{\omega} \omega_{0}(\boldsymbol{r})+g_{\rho} \tau_{3} \rho_{0}(\boldsymbol{r})+e A_{0}(\boldsymbol{r}),
$$

and a magnetic potential

$$
\boldsymbol{V}(\boldsymbol{r})=g_{\omega} \boldsymbol{\omega}(\boldsymbol{r})+g_{\rho} \tau_{3} \boldsymbol{\rho}(\boldsymbol{r})+e \boldsymbol{A}(\boldsymbol{r}) .
$$

The last term breaks time-reversal symmetry and induces currents. For example, time-reversal symmetry is broken when the time-reversed orbitals are not occupied pairwise; this takes place in odd-mass nuclei [29]. However, nuclear magnetism [28], i.e. currents and time-odd mean fields, plays no role in the studies of ground states and fission barriers in even-even nuclei. Thus, magnetic potential is neglected in the present RHB calculations.

In order to avoid the uncertainties connected with the definition of the size of the pairing window [73], we use the separable form of the finite range Gogny pairing interaction introduced by Tian et al [74]. Its matrix elements in $r$-space have the form

$$
\begin{aligned}
& V\left(\boldsymbol{r}_{1}, \boldsymbol{r}_{2}, \boldsymbol{r}_{1}^{\prime}, \boldsymbol{r}_{2}^{\prime}\right)= \\
& \quad=-G \delta\left(\boldsymbol{R}-\boldsymbol{R}^{\prime}\right) P(r) P\left(r^{\prime}\right) \frac{1}{2}\left(1-P^{\sigma}\right)
\end{aligned}
$$

with $\boldsymbol{R}=\left(\boldsymbol{r}_{1}+\boldsymbol{r}_{2}\right) / 2$ and $\boldsymbol{r}=\boldsymbol{r}_{1}-\boldsymbol{r}_{2}$ being the center of mass and relative coordinates. The form factor $P(r)$ is of Gaussian shape

$$
P(r)=\frac{1}{\left(4 \pi a^{2}\right)^{3 / 2}} e^{-r^{2} / 4 a^{2}}
$$

The two parameters $G=728 \mathrm{MeVfm}^{3}$ and $a=0.644$ $\mathrm{fm}$ of this interaction are the same for protons and neutrons and have been derived in Ref. [74] by a mapping of the ${ }^{1} \mathrm{~S}_{0}$ pairing gap of infinite nuclear matter to that of the Gogny force D1S [75]. This pairing provides a reasonable description of pairing properties in the actinides (see Refs. $[39,76,77]$ ) and has been used in our previous studies of different phenomena in actinides, super- and hyperheavy nuclei in Refs. [39, 48, 59, 61, 65, 66].

The truncation of the basis is performed in such a way that all states belonging to the major shells up to $N_{F}=20$ fermionic shells for the Dirac spinors and up to $N_{B}=20$ bosonic shells for the meson fields are taken into account. Note that the latter applies only to the NL3* and DD-ME2 functionals which contain meson exchange. As follows from investigation of Refs. [39, 78] this truncation of basis provides sufficient numerical accuracy.

The calculations are performed in the following way: 

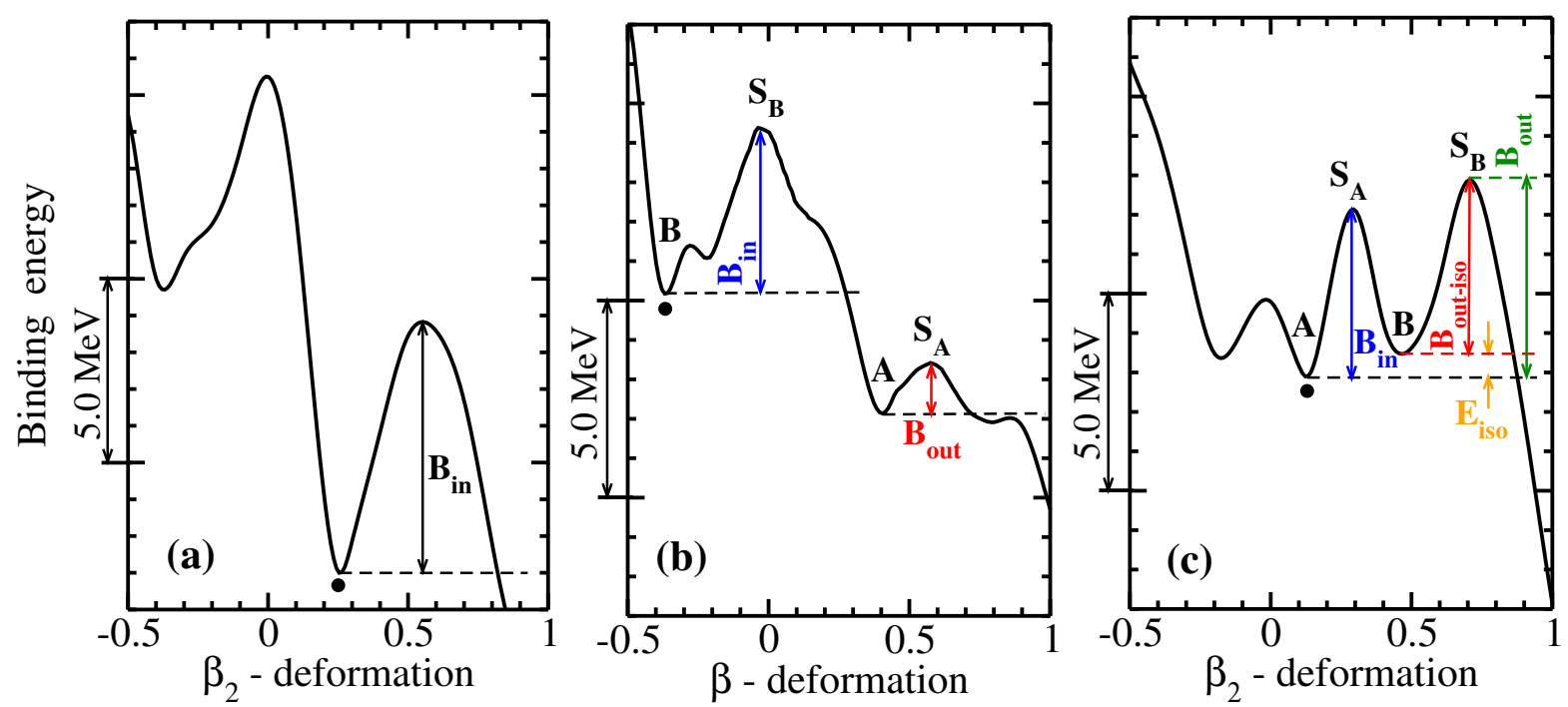

FIG. 2. Schematic illustration of different types of deformation energy curves and the selection of respective ground states (see text for details). Local minima are labelled by the letters $\mathrm{A}$ and $\mathrm{B}$ and the saddle points of respective fission barriers by $\mathrm{S}_{\mathrm{A}}$ and $\mathrm{S}_{\mathrm{B}}$. Solid circles indicate the minima selected as the ground states. The heights of inner and outer fission barriers with respect of corresponding minima (shown by dashed lines) are indicated by $B_{\text {in }}$ and $B_{\text {out }}$. $B_{\text {out-iso }}$ is the height of outer fission barrier with respect of fission isomer.

- Reflection-symmetric constrained axial RHB calculations (further RS-RHB) are performed for each nucleus and the potential energy curve is defined in a large deformation range from $\beta_{2}=-1.0$ up to $\beta_{2}=1.6$ in step of $\Delta \beta_{2}=0.05$ by means of the constraint on the quadrupole moment $q_{20}$. The calculations are performed by successive diagonalizations using the method of quadratic constraints [79]. The parallel version of computer code allows simultaneous calculations for a significant number of nuclei and deformation points in each nucleus. For each nucleus, we minimize

$$
E_{\text {quad }}=E_{R H B}+C_{20}\left(\left\langle\hat{Q}_{20}\right\rangle-q_{20}\right)^{2}
$$

where $E_{R H B}$ is the total energy and $\left\langle\hat{Q}_{20}\right\rangle$ denotes the expectation value of the mass quadrupole operator,

$$
\hat{Q}_{20}=2 z^{2}-x^{2}-y^{2}
$$

$q_{20}$ is the constrained value of the multipole moment, and $C_{20}$ the corresponding stiffness constant [79]. In order to provide the convergence to the exact value of the desired multipole moment we use the method suggested in Ref. [80]. Here the quantity $q_{20}$ is replaced by the parameter $q_{20}^{e f f}$, which is automatically modified during the iteration in such a way that we obtain $\left\langle\hat{Q}_{20}\right\rangle=q_{20}$ for the converged solution. This method works well in our constrained calculations.

- In addition, reflection-asymmetric (octupole deformed) constrained axial RHB calculations (further RA-RHB) are performed in discussed below cases using parallel version of the code developed in Ref. [48]. In these calculations, the constraint

$$
E_{\text {quad }}+C_{30}\left(\left\langle\hat{Q}_{30}\right\rangle-q_{30}\right)^{2}
$$

is employed in addition to the constraint on quadrupole moment (see Eq. (9)). Here $\left\langle\hat{Q}_{30}\right\rangle$ denotes the expectation value of the mass octupole operator

$$
\hat{Q}_{30}=z\left(2 z^{2}-3 x^{2}-3 y^{2}\right) .
$$

Note that we also fix the (average) center of mass of the nucleus at the origin with the constraint

$$
\left\langle\hat{Q}_{10}\right\rangle=0
$$

on the center-of-mass operator $\hat{Q}_{10}$ in order to avoid a spurious motion of the center of mass. In the present paper, reflection asymmetric RHB calculations have been performed for the ground states of the nuclei not covered in previous systematic studies of octupole deformation in CDFT (see Refs. $[48,61])$. We have not found any additional (as compared with those given in Refs. [48, 61]) nuclei which possess octupole deformation in the ground state. So full information on the octupole deformation of the ground states can be found in these references. The information (which follows from Refs. $[48,61])$ about the gain in binding energy due to octupole deformation at the ground state and its impact on ground state quadrupole deformation and fission barrier heights is fully taken into account in the present paper. In addition, RA-RHB calculations have been performed in some nuclei in order 

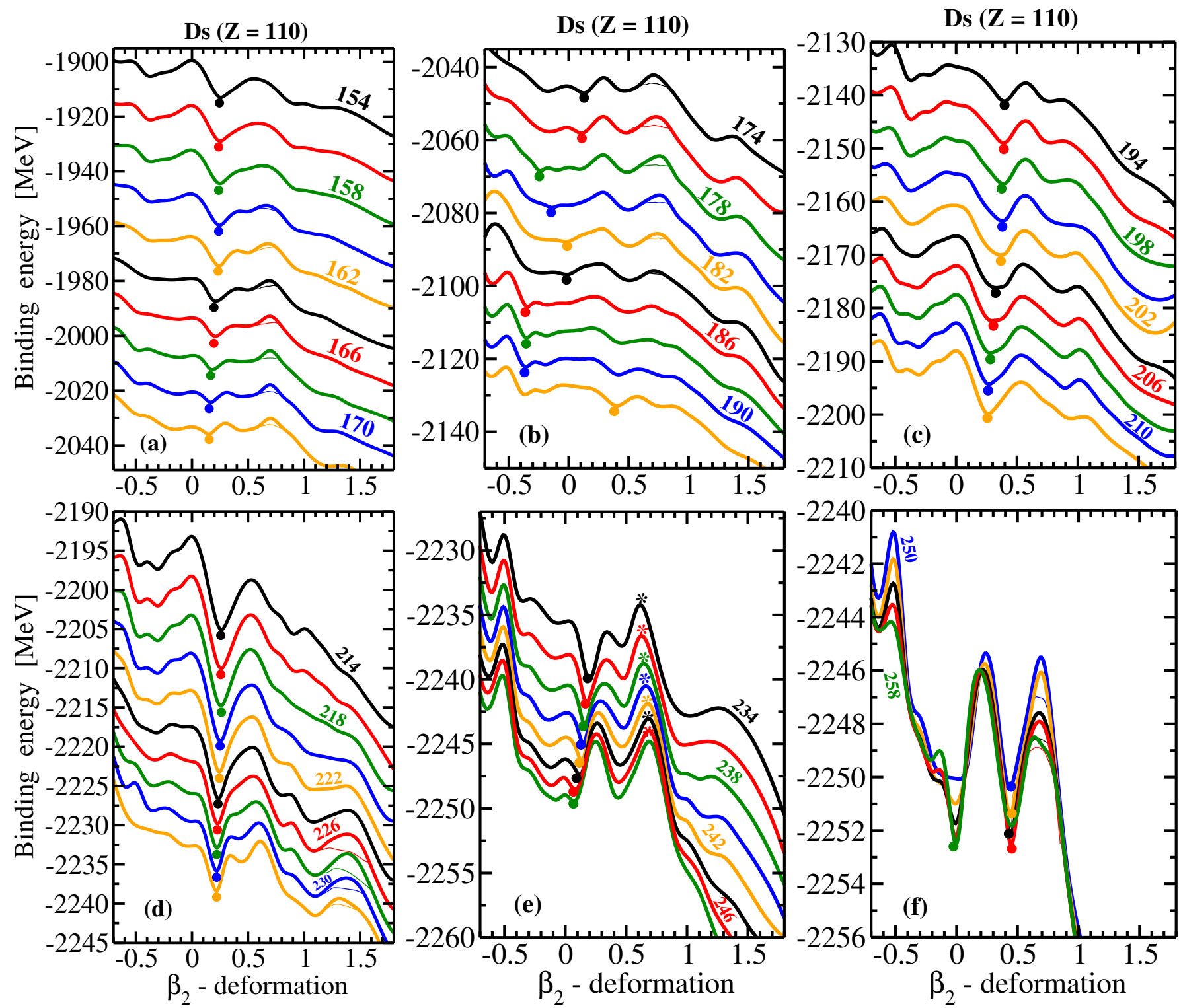

FIG. 3. Deformation energy curves obtained in axial RHB calculations with DDPC1 functional for the Ds isotopes. The isotopes are indicated by respective neutron numbers. Thick and thin solid lines are used for the RS-RHB and RA-RHB results, respectively. The results of the RA-RHB calculations are shown only in the deformation range in which they are lower in energy than the RS-RHB ones. Solid circles indicate the ground states and the asterisks denote the saddles of outer fission barriers which are not affected by octupole deformation. Blue, orange, black, red and green lines are used to indicate neutron numbers the last digits of which are $0,2,4,6$, and 8 , respectively. Note that the energy on the vertical axis spans different ranges in different panels.

to define the heights of outer fission barriers (see the discussion below for more details).

The charge quadrupole and octupole moments are defined as

$$
\begin{aligned}
& Q_{20}=\int d^{3} r \rho(\boldsymbol{r})\left(2 z^{2}-r_{\perp}^{2}\right), \\
& Q_{30}=\int d^{3} r \rho(\boldsymbol{r}) z\left(2 z^{2}-3 r_{\perp}^{2}\right)
\end{aligned}
$$

with $r_{\perp}^{2}=x^{2}+y^{2}$. In principle these values can be directly compared with experimental data. However, it is more convenient to transform these quantities into dimensionless deformation parameters $\beta_{2}$ and $\beta_{3}$ using the relations

$$
\begin{aligned}
Q_{20} & =\sqrt{\frac{16 \pi}{5}} \frac{3}{4 \pi} Z R_{0}^{2} \beta_{2}, \\
Q_{30} & =\sqrt{\frac{16 \pi}{7}} \frac{3}{4 \pi} Z R_{0}^{3} \beta_{3}
\end{aligned}
$$

where $R_{0}=1.2 A^{1 / 3}$. These deformation parameters are more frequently used in experimental works 

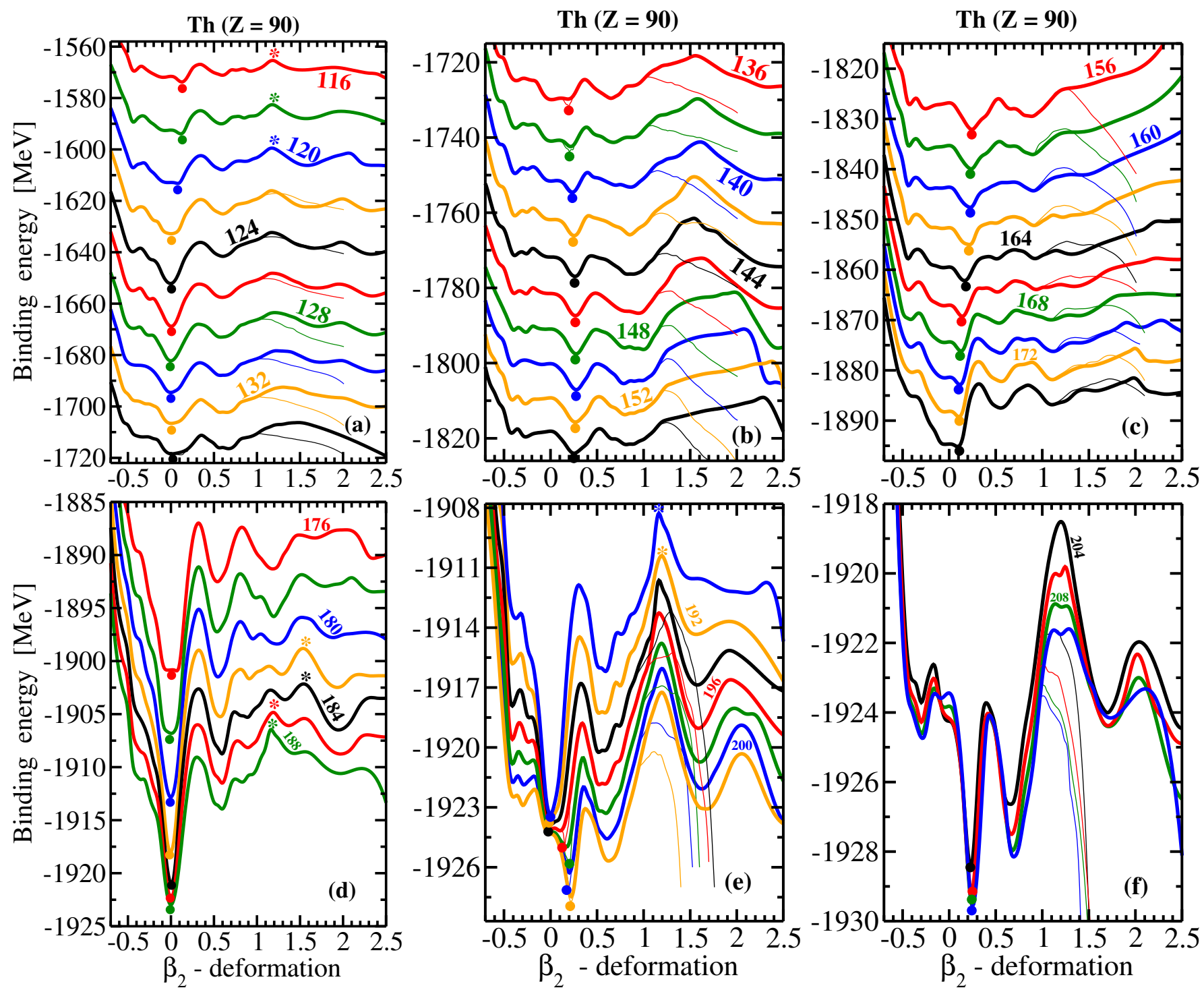

FIG. 4. The same as Fig. 3 but for the Th isotopes. Note that the deformation range has been extended on horizontal axis as compared with Fig. 3. In order to save computational time the RA-RHB calculations have been carried out only up to $\beta_{2}=2.0$.

than quadrupole and octupole moments. In addition, the potential energy surfaces (PES) are plotted in this manuscript in the $\left(\beta_{2}, \beta_{3}\right)$ deformation plane.

Because of different patterns of deformation energy curves (see Figs. 3 and 4), a special care is used when assigning a specific minimum to the ground state. A basic rule in this process is the assumption that local minimum surrounded by the barrier, the height of which is less than $2 \mathrm{MeV}$, is considered as extremely unsta$b^{4}$. The procedure of the selection of the ground state is discussed below. The situation shown in Fig. 2a is the

\footnotetext{
${ }^{4}$ This low fission barrier of $2 \mathrm{MeV}$ or less would translate into a high penetration probability for spontaneous fission so that such minima are expected to be extremely unstable. In addi-
}

simplest one: single-humped (inner) fission barrier acts against the fission into two fragments and when $\mathrm{B}_{\text {in }}>2$ $\mathrm{MeV}$ the assignment of the normal-deformed prolate minimum to the ground state is straightforward. It changes if the height of this fission barrier decreases and becomes smaller than $2 \mathrm{MeV}$ (see Fig. 2b). Then highly-deformed oblate minimum B becomes a ground state; it has larger and broader fission barrier as compared with minimum A. Thus, it is expected that this ground state will live significantly longer than the state associated with minimum

tion, the inclusion of octupole deformation (or triaxial deformation in some nuclei [78]) in the case of superdeformed minima surrounded by such low fission barriers could either completely eliminate or substantially reduce them (see Refs. [66, 78, 81]). 


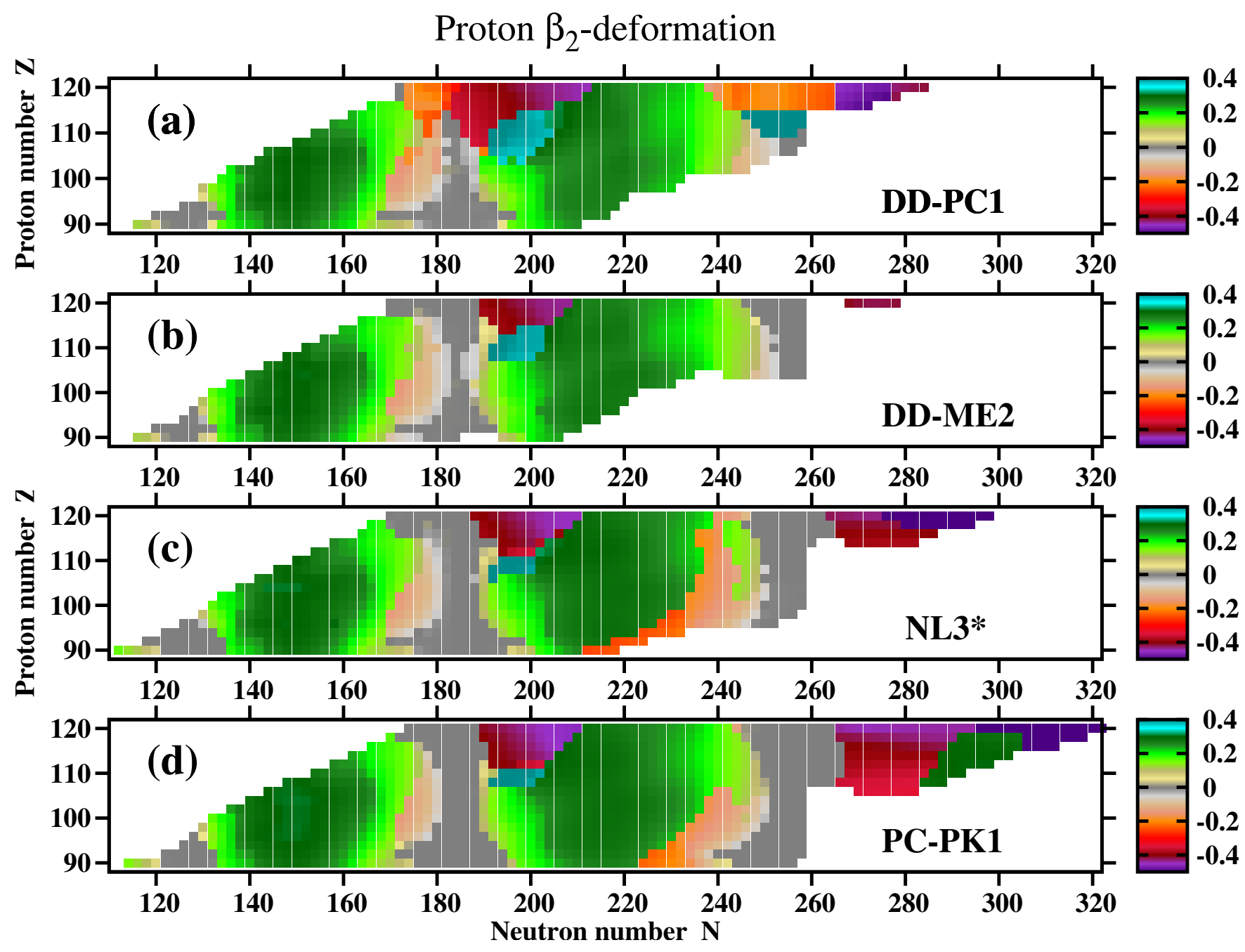

FIG. 5. Proton quadrupole deformations $\beta_{2}$ obtained in the RS-RHB and RA-RHB calculations with indicated CEDFs (see Refs. [48,61] for the details of the regions of octupole deformation). Note that last bound $Z=120$ nucleus appears at $N=324$ in the calculations with the CEDF PC-PK1 (see Table III below); for simplicity it is not shown in panel (d).

A.

More complex situation involving two humped fission barrier is shown in Fig. 2(c). If $B_{\text {out-iso }}<2 \mathrm{MeV}$, then the minimum $\mathrm{B}$ corresponding to fission isomer is considered extremely unstable and the minimum A is associated with the ground state. Note that in some cases the minimum $\mathrm{B}$ can be lower in energy than minimum A. If that is the case and if $B_{\text {out-iso }}>2 \mathrm{MeV}$ then the minimum B is considered as the ground state. Note that the heights of outer fission barriers are frequently lowered when octupole deformation is included in the calculations $[63,69,78,81-83]$. Thus, if $B_{\text {out-iso }}>2$ $\mathrm{MeV}$ in RS-RHB calculations, we perform RA-RHB calculations in the region of the $\left(\beta_{2}, \beta_{3}\right)$ plane covering the minimum $\mathrm{B}$ and the saddle of outer fission barrier on the grid with the steps of $\Delta \beta_{2}=\Delta \beta_{3}=0.05$. This allows to establish whether minimum $\mathrm{B}$ could be considered as relatively stable or unstable. Similar calculations are also performed in the cases when $\mathrm{B}_{\mathrm{in}}<\mathrm{B}_{\text {out }}$ in the RS-RHB calculations. This is because we consider only the height of the primary (highest) fission barrier (PFB) in Sec. V in the case of doubly-humped structure of the barrier to minimize the computational cost. Note that the calculations leading to the definition of the fission path and the saddle point in the RA-RHB code are by roughly two orders of magnitude more time consuming than those in the RS-RHB code.

The procedure outlined above takes into account potential stability of the nuclei in respective energy minima with respect of fission and it is especially important in superheavy nuclei some local minima of which are characterized by small fission barriers (see Fig. 3 ). Note that after defining the minimum corresponding to the ground state, the RS-RHB and RA-RHB calculations without constraint are performed in it and precise binding energy and equilibrium of the ground state is determined. In addition, the height(s) of the fission barrier(s) is(are) defined. 

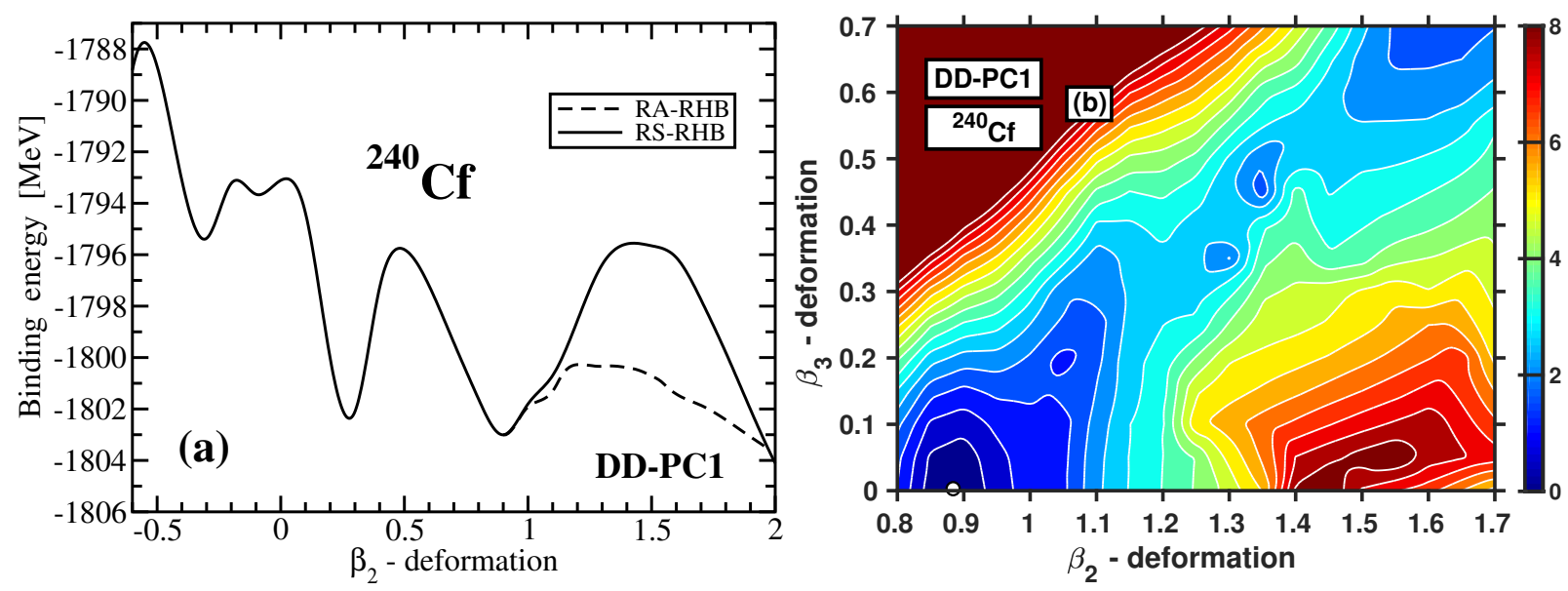

FIG. 6. (a) Deformation energy curves obtained in axial RS-RHB calculations with the CEDF DD-PC1 for the ${ }^{240} \mathrm{Cf}$ nucleus. (b) Potential energy surface in the $\left(\beta_{2}, \beta_{3}\right)$ plane obtained in the RA-RHB calculations. Extremely superdeformed minimum is indicated by open white circle.

In the calculations with the PC-PK1 and NL3* functionals there are two small islands of the nuclei located in the $Z \approx 114-118, N \approx 238-240$ and $Z \approx 106-110, N \approx$ $190-194$ regions in which calculated deformation energy curves reveal several local minima (somewhat similar to the deformation energy curves shown at the bottom of Fig. 3b). However, all these minima are surrounded by very low fission barriers with the heights smaller than 2.0 $\mathrm{MeV}$. Moreover, many of these minima have even lower heights of respective fission barriers (on the level of 1.0 $\mathrm{MeV}$ or smaller). These nuclei are expected to be unstable against fission and in principle it does not matter which of the calculated fission barriers is used. For these nuclei, we select the ground state guided by the flow of the $\beta$-decays in the r-process: the selected local minimum (and thus the corresponding ground state deformation and fission barrier height) in the $(Z, N)$ nucleus has the deformation closest to the one of the well established ground state in the $(Z-2, N+2)$ nucleus.

So far all existing global calculations of the fission barriers for the r-process simulations have been performed in non-relativistic models. These include the calculations within the FRDM [18, 84], the TF and ETFSI$\mathrm{Q}$ approaches $[15,19]$, the HFB approaches with different Skyrme functionals [21, 22, 85], Gogny D1M* [23] and BCPM [18] EDFs. Because of their global character, all these calculations are restricted to axial symmetry. We also assume axial symmetry in our calculations because triaxial $\mathrm{RMF}+\mathrm{BCS}$ and $\mathrm{RHB}$ calculations (see Refs. $[47,62,66,78,86])$ are too time consuming to be performed on a global scale. Note also that dynamical correlations are not taken into account in our calculations of fission barriers because of the reasons discussed in Appendix A.

\section{GROUND STATE PROPERTIES}

The distributions of calculated proton deformations $\beta_{2}$ in the $(Z, N)$ plane obtained with four employed CEDFs are shown in Fig. 5. The width of the gray region (the gray color corresponds to spherical and near-spherical shapes) along a specific magic number corresponding to a shell closure indicates the impact of this shell closure on the structure of neighboring nuclei. Note that proton and neutron shell gaps act simultaneously in the vicinity of doubly magic spherical nuclei. Thus, the effect of a single gap is more quantifiable away from these nuclei. One can see that neutron $N=184$ and $N=258^{5}$ spherical shell gaps have a pronounced impact on calculated deformations while the impact of the proton $Z=120$ spherical shell closure is limited to the $N \sim 170-184$ nuclei (see Refs. [59, 90] for examples of their size dependence on the functional). In addition, as illustrated in Ref. [86] on the example of the PC-PK1 functional, the inclusion of the correlations beyond mean field washes out the impact of the $Z=120$ shell closure and leads to oblate deformed ground states in the majority of the $Z=120$ nuclei with $N=172-186$. The predictions of the DDPC1 functional differs substantially from other CEDFs: the impact of above mentioned shell closures are substantially reduced in it and as a consequence the regions with $Z \sim 120, N \sim 184$ and $Z \sim 120, N \sim 258$ are dominated by oblate ground states contrary to spherical states in other functionals. Note that this functional provides the

\footnotetext{
${ }^{5}$ Note that appreciable $N=258$ spherical shell gap appears also in the calculations of some superheavy nuclei with other CEDFs such as G1, G2 [87], NL3 [87-89], NL-Z2 [88], NL1, NLSH, TM1, TW99, DD-ME1, PK1, and PK1R [89]. However, these calculations are restricted to spherical shapes and thus it is not clear how large is the impact of this gap on ground state deformations in the region near the $N=258$ line.
} 


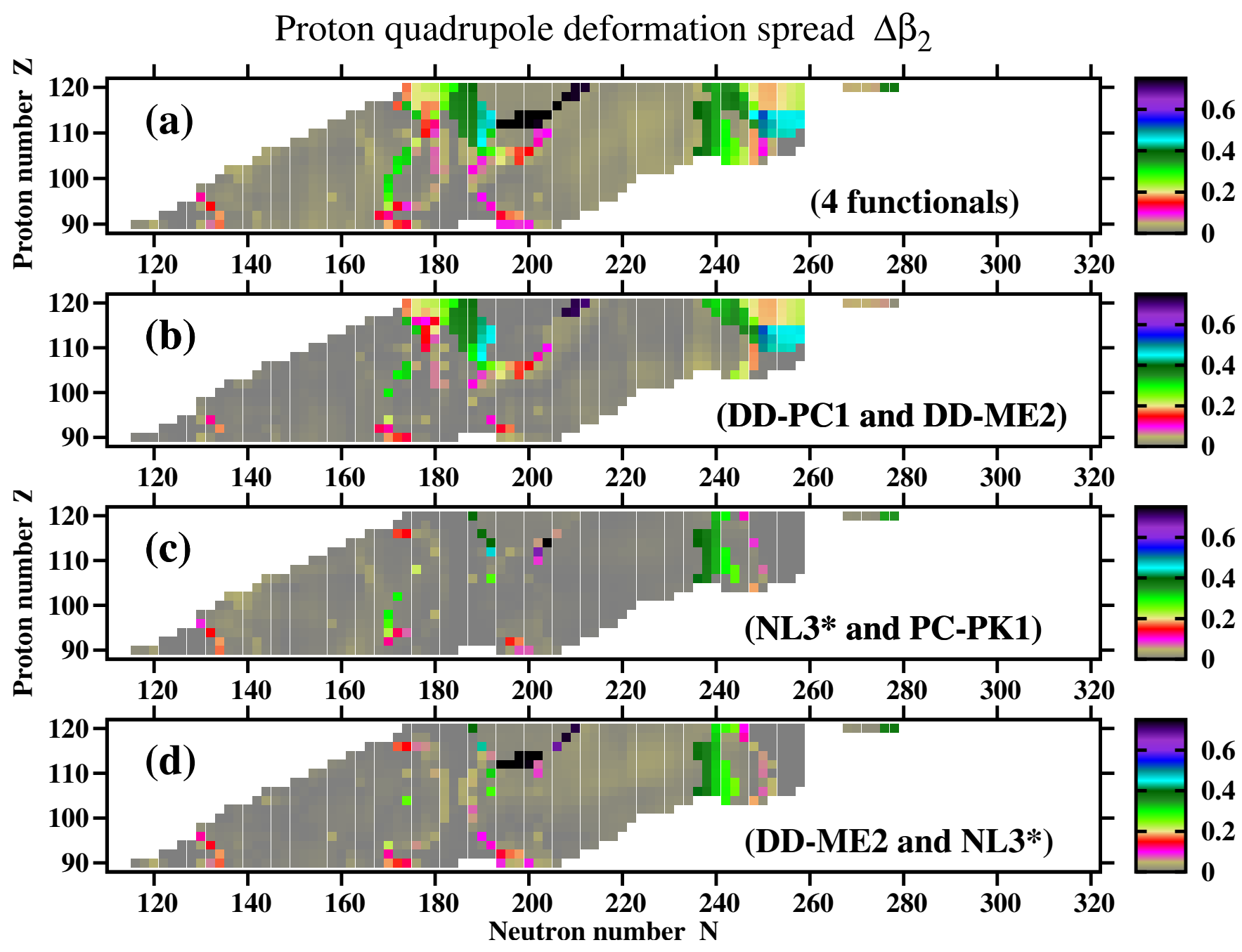

FIG. 7. Proton quadrupole deformation spreads $\Delta \beta_{2}(Z, N)$ as a function of proton and neutron number. $\Delta \beta_{2}(Z, N)=$ $\left|\beta_{2}^{\max }(Z, N)-\beta_{2}^{\min }(Z, N)\right|$, where $\beta_{2}^{\max }(Z, N)$ and $\beta_{2}^{\min }(Z, N)$ are the largest and smallest proton quadrupole deformations obtained with four employed CEDF for the $(Z, N)$ nucleus.

best global description of experimental binding energies (see Ref. [39]). This, however, does not guarantee that it will outperform other functionals in the description of physical observables of interest in the region of superheavy nuclei (see Table I in Ref. [59]).

The calculations reveal a number of nuclei scattered across the part of nuclear chart under study which have extremely superdeformed (ESD) minimum with $\beta_{2} \sim 1.0$ located at lower energy than normal-deformed minimum (see Fig. 6a and Table I). In these nuclei the ESD minimum is surrounded by outer fission barrier the height of which exceeds $2.0 \mathrm{MeV}$ (being typically in the range of $2.0-3.0 \mathrm{MeV}$ ) in the RA-RHB calculations (see Fig. $6 \mathrm{~b}$ and Table I). Although the fission barrier is low, some of these ESD minima could be potentially stabilized against fission for physically sufficient time because of broad fission barrier in the $\left(\beta_{2}, \beta_{3}\right)$ plane. If that would be a case, they would represent the ground states. However, they are not included into Fig. 5 because of the following reasons. First, there are significant theoretical uncertainties in the predictions of fission barriers (see present paper and Refs. $[47,78]$ ) as well as in relative energies of the minima with different deformations [73]. Second, only few nuclei in the $Z \approx 100, N \approx 230$ region could be potentially extremely supedeformed in the ground states (see Table I). However, the flow of matter in the r-process between two nuclei with drastically different deformations of the ground states will be most likely significantly suppressed because of considerable differences in the wave functions of these ground states. Thus, it will proceed mostly along the dominant deformation of the ground states in the region, namely, normal deformation, even if such states are excited in energy in a few nuclei. Third, the majority of the nuclei in Table I are neutron poor $Z \approx 102$ nuclei which do not play a role in the r-process. There are experimental data on the ${ }^{240,242} \mathrm{Cf},{ }^{246} \mathrm{Fm}$ and ${ }^{254} \mathrm{Rf}$ nuclei but only for their ground states [91]. At present, these data 


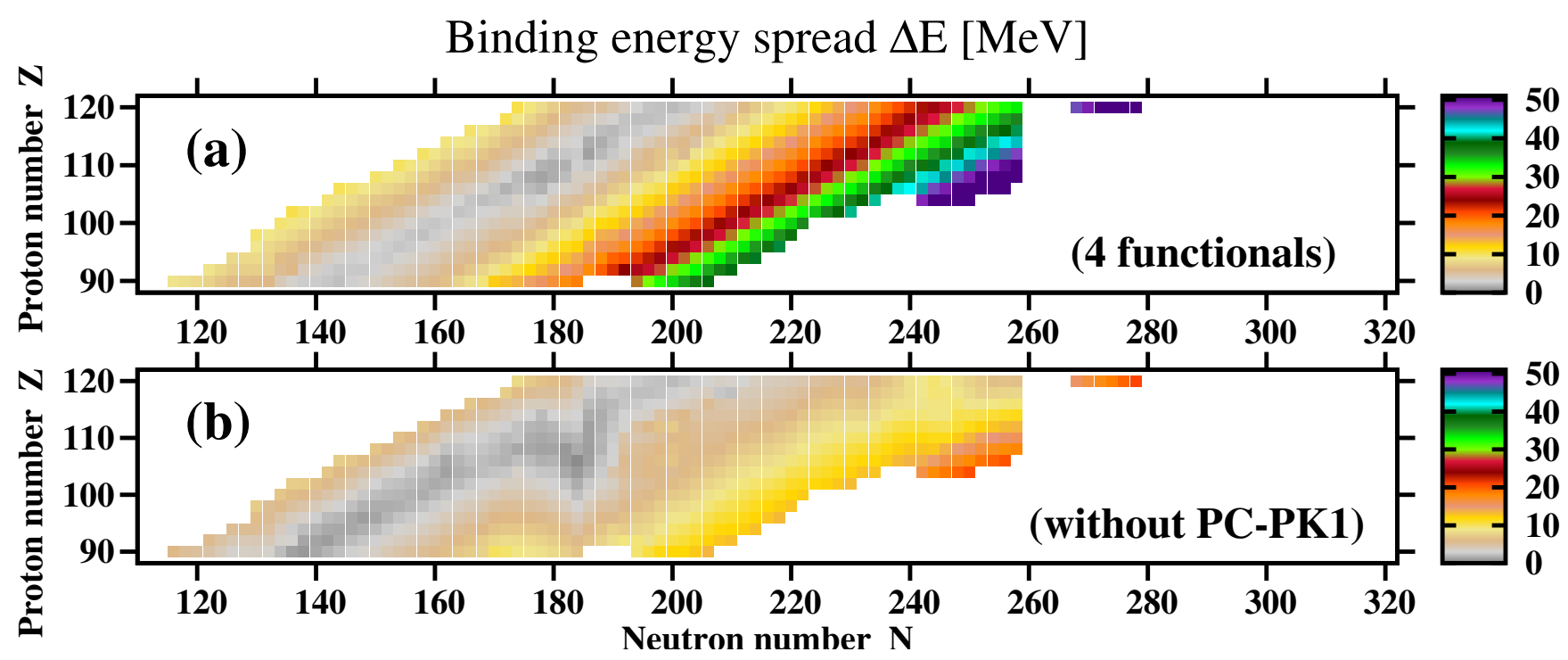

FIG. 8. The binding energy spreads $\Delta E(Z, N)$ as a function of proton and neutron number. $\Delta E(Z, N)=\mid E_{\max }(Z, N)-$ $E_{\min }(Z, N) \mid$, where $E_{\max }(Z, N)$ and $E_{\min }(Z, N)$ are the largest and smallest binding energies obtained with employed set of CEDFs for the $(Z, N)$ nucleus. Upper panel shows the binding energy spreads for four employed functionals, while the bottom one the spreads for a set of functionals in which PC-PK1 is excluded.

do not allow to define the deformations of the ground states. However, since it has been obtained in the reactions (such as $\alpha$-decay, $\beta$-decay, electron capture and the reactions on spherical $\mathrm{Pb}$ isotopes) which do not favor significant shape changes, these ground states are most likely normal-deformed. More detailed and focused experimental studies are needed in order to see whether ESD states exist in such nuclei.

TABLE II. Selected properties of symmetric nuclear matter at saturation: the incompressibility $K_{0}$, the symmetry energy $J$ and its slope $L_{0}$. Top four lines show the values for indicated CEDFs, while bottom two lines show two sets (SET2a and SET2b) of the constraints on the experimental/empirical ranges for the quantities of interest defined in Ref. [51]. The CEDF values which are located outside the limits of the SET2b constraint set are shown in bold.

\begin{tabular}{|c|c|c|c|}
\hline CEDF & $K_{0}[\mathrm{MeV}]$ & $J[\mathrm{MeV}]$ & $L_{0}[\mathrm{MeV}]$ \\
\hline 1 & 2 & 3 & 4 \\
\hline NL3 ${ }^{*}[43]$ & 258 & $\mathbf{3 8 . 6 8}$ & $\mathbf{1 2 2 . 6}$ \\
DD-ME2 [42] & 251 & 32.40 & 49.4 \\
DD-PC1 [44] & 230 & 33.00 & 68.4 \\
PC-PK1 [50] & 238 & $\mathbf{3 5 . 6}$ & $\mathbf{1 1 3}$ \\
SET2a & $190-270$ & $25-35$ & $25-115$ \\
SET2b & $190-270$ & $30-35$ & $30-80$ \\
\hline
\end{tabular}

The spreads of theoretical predictions in quadrupole deformations $\beta_{2}$ obtained with four employed functionals are summarized in Fig. 7(a). The largest spread of $\Delta \beta_{2} \approx 0.7$ is visible along the line of $N / Z \approx 1.81$ which starts at $Z=104$. This corresponds to the boundary of the transition from oblate to prolate shapes the exact position of which in the $(Z, N)$ plane is functional dependent (see Fig. 5). It is defined by the underlying single-particle structure at prolate and oblate shapes as well as to a degree by the heights of outer fission barriers (see the rules for the definition of the ground states described in Sec. II). Second region of the largest spreads in $\Delta \beta_{2}$ is located along the $N \approx 184$ line starting from $Z \approx 100$ and extending up to $Z=120$. Third region is located along the $Z=120$ line from proton-drip line up to $N \approx 188$. These two regions of large spreads in calculated quadrupole deformation emerge from the differences in the predictions of ground state deformations (see Fig. 5) which in turn can be traced back to the sizes of the $Z=120$ and $N=184$ spherical shell closures and the densities of the single-particle states in their vicinities (see Ref. [59]). The last region of large theoretical uncertainties is located between $N \approx 236$ and $N=258$. In the region around $N \approx 236$ these theoretical uncertainties are mostly due to the uncertainties in the predictions of the boundary of the transition from prolate to oblate shapes. For higher $N$ values, large $\Delta \beta_{2}$ values emerge from the transition from prolate or oblate shapes to spherical ones and to a large degree are defined by the uncertainties in the prediction of the size of the $N=258$ spherical shell closure (see Fig. 6d in Ref. [90]) and single-particle densities in its vicinity. With few exceptions theoretical uncertainties in the predictions of ground state deformations in the part of nuclear chart outside of above discussed regions are very small (see Fig. 7(a)).

It is important to understand to what extent the predictions of the ground state deformations and related theoretical uncertainties in these predictions are dependent on nuclear matter properties of employed CEDFs. All employed CEDFs have the density $\rho_{0}$ and the energy per particle $E / A$ at the saturation of symmetric nuclear 


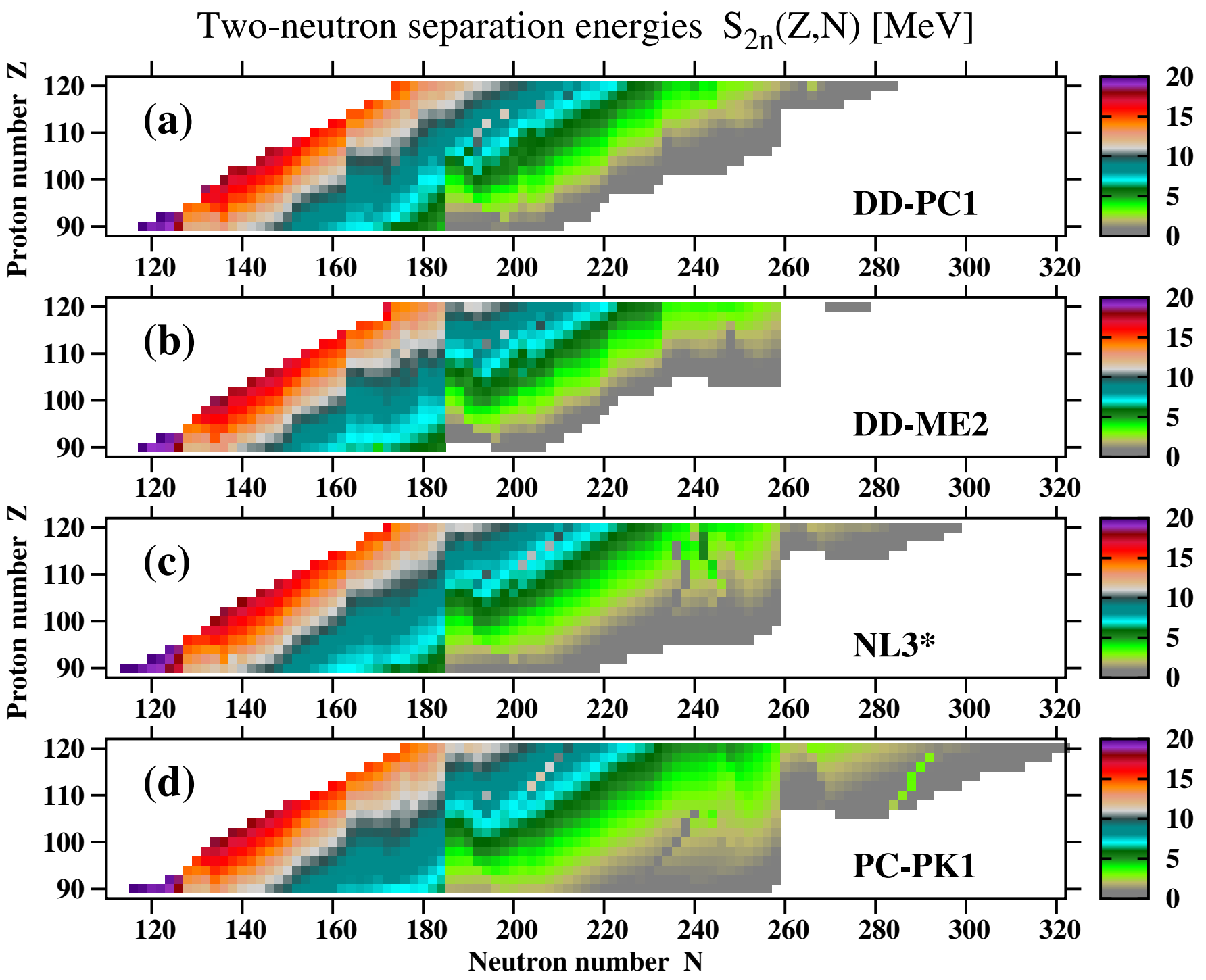

FIG. 9. Two-neutron separation energies $S_{2 n}(Z, N)$ obtained in the RHB calculations with indicated CEDFs.

matter (SNM) which are very close to each other and to empirical estimates (see Table III in Ref. [49]). Thus, the impact of only selected SNM properties listed in Table II on the ground state deformations are discussed below. Let start from the consideration of the predictions by the pair of the functionals DD-PC1 and DD-ME2. Their SNM properties such as incompressibility $K_{0}$, the symmetry energy $J$ and its slope $L_{0}$ are close to each other and are located within the SET2b constraints on experimental/empirical ranges for physical observables of interest (see Table II). Despite that this pair of the functionals gives the largest contribution into the spreads $\Delta \beta_{2}$ (compare panels (b) and (a) of Fig. 7). On the contrary, the pairs of the functionals PC-PK1 and NL3* (which have $J$ and $L_{0}$ values located outside the SET2b constraint range [see Table II]) as well as NL3* and DD-ME2 (which have drastically different values of the $J$ and $L_{0}$ parameters [see Table II]) have (with very few exceptions) very sim- ilar predictions for the ground state deformations across the part of nuclear chart under study. These exceptions are related to some differences in the predictions of the boundaries between oblate and prolate shapes as well as between prolate and spherical shapes.

These results for ground state deformations together with the analysis of the results for binding energies and charge radii of the $Z \leq 104$ nuclei presented in Ref. [49] strongly indicate that

- the major source of the uncertainties in the predictions of ground state deformations is related to local differences in underlying single-particle structure and, in particular, to the size of spherical $Z=120$ and $N=184$ and 258 shell closures and the densities of the single-particle states in their vicinities,

- strict enforcement of the limits on the nuclear mat- 


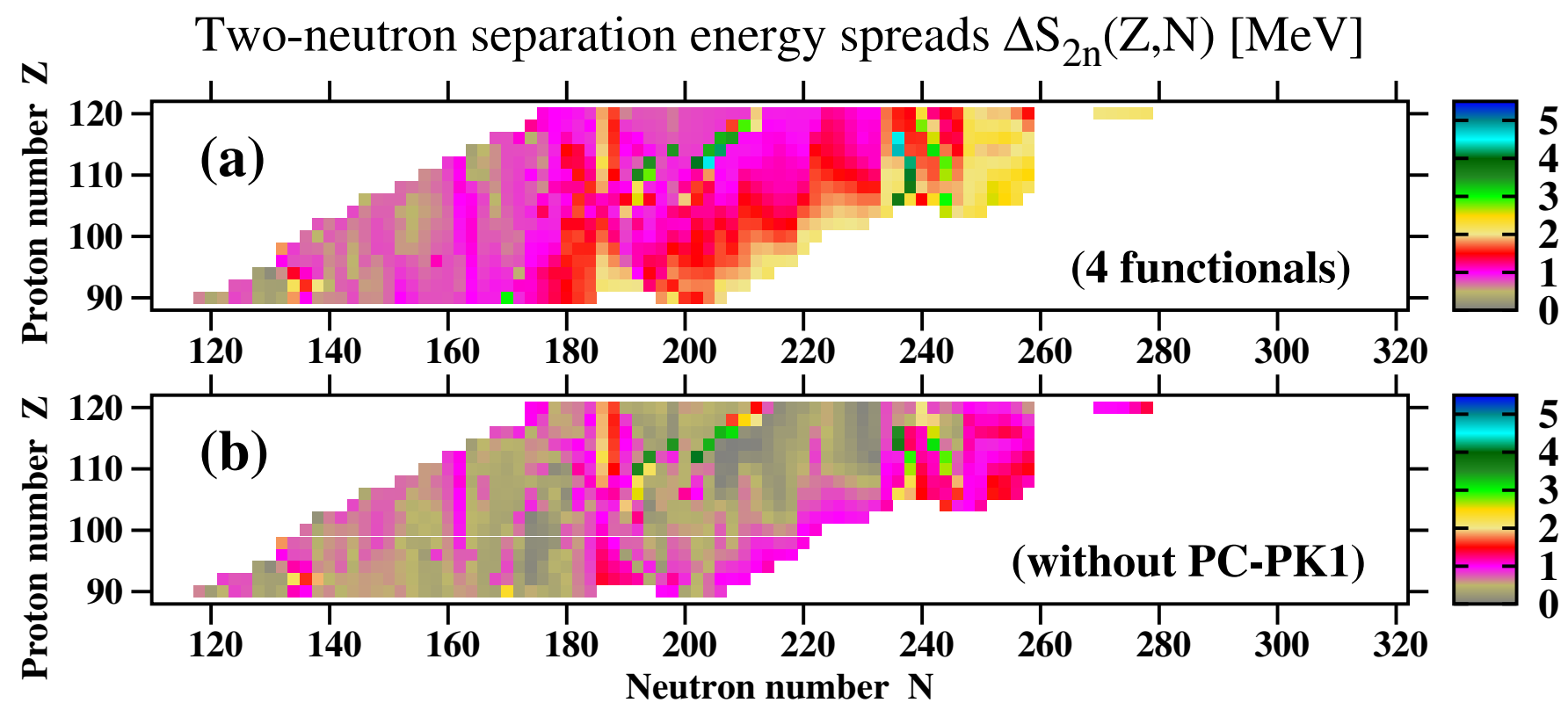

FIG. 10. The $S_{2 n}$ spreads $\Delta S_{2 n}(Z, N)$ as a function of proton and neutron number. $\Delta S_{2 n}(Z, N)=\left|S_{2 n}^{\max }(Z, N)-S_{2 n}^{\min }(Z, N)\right|$, where $S_{2 n}^{\max }(Z, N)$ and $S_{2 n}^{\min }(Z, N)$ are the largest and smallest $S_{2 n}$ values obtained with four employed CEDFs for the $(Z, N)$ nucleus.

ter properties defined in Ref. [51] will not necessary lead to the functionals with good description of ground and excited state properties and will not reduce theoretical uncertainties in the description of physical observables of interest in high- $Z$ and/or neutron-rich nuclei.

Table III compares the positions of the two-proton and two-neutron drip lines obtained in the RHB calculations with the NL3* and PC-PK1 functionals; the results for the DD-PC1 and DD-ME2 functionals could be found in Table IV of Ref. [39]. One can see that the two-proton drip lines are very similar in these two functionals; they differ by no more than four neutrons. This is in line with earlier observations that theoretical uncertainties in the predictions of the position of two-proton drip line are relatively small (see Ref. [93] and Sec. VII in Ref. [39]). Note that two-proton drip lines in the isotopic chains of interest obtained with PC-PK1 are very close to those obtained with DD-PC1 (compare Table III in the present article with Table IV of Ref. [39]).

Among the considered CEDFs the PC-PK1 functional provides the most neutron-rich two-neutron drip line and the NL3* provides the second most neutron-rich twoneutron drip lines (compare Table III in the present article with Table IV of Ref. [39] and see Sec. VIII in Ref. [39]). All employed functionals reveal the presence of the shell closure at $N=258$ (see Fig. 6d in Ref. [90]). The size of this gap is almost the same in the NL3* and PCPK1 functionals, but (i) it is shifted down in energy by $\approx 400 \mathrm{keV}$ for PC-PK1 as compared with NL3* and (ii) high- $j$ intruder orbitals $1 k_{15 / 2}$ and $2 i_{13 / 2}$, which have a significant impact on the position of neutron drip line
TABLE III. Two-proton and two-neutron drip lines predicted by the NL3* and PC-PK1 ${ }^{\mathrm{a}}$ functionals (see Fig. 1 for graphical representation of drip lines). Neutron numbers (columns 2-5) corresponding to these drip lines are given for each even proton number $Z$ (column 1). An asterisk at a neutron number at the two-neutron drip line indicates isotope chains with additional two-neutron binding at higher $N$ values (peninsulas).

\begin{tabular}{|c|c|c|c|c|}
\hline Proton & \multicolumn{2}{|c|}{ Two-proton drip line } & \multicolumn{2}{c|}{ Two-neutron drip line } \\
number $Z$ & NL3 & PCPK1 & NL3 & PCPK1 \\
\hline 1 & 2 & 3 & 4 & 5 \\
\hline 90 & 112 & 114 & 218 & 256 \\
92 & 118 & 122 & 224 & 258 \\
94 & 122 & 126 & 232 & 258 \\
96 & 126 & 128 & 252 & 258 \\
98 & 130 & 130 & 256 & 258 \\
100 & 132 & 132 & 258 & 258 \\
102 & 134 & 136 & 258 & 258 \\
104 & 138 & 140 & 258 & 258 \\
106 & 142 & 144 & 258 & $258^{*}$ \\
108 & 146 & 148 & 258 & 288 \\
110 & 150 & 154 & 258 & 292 \\
112 & 154 & 158 & 258 & 298 \\
114 & 158 & 162 & $262^{*}$ & 302 \\
116 & 162 & 166 & 286 & 312 \\
118 & 166 & 170 & 294 & 318 \\
120 & 170 & 174 & 298 & 324 \\
\hline
\end{tabular}

a The analysis of Ref. [92] performed within the relativistic continuum Hartree-Bogoliubov theory and PC-PK1 functional leads to somewhat different predictions for the position of the two-neutron drip line for some isotopic chains as compared with our results. This is a consequence of the neglect of deformation effects in Ref. [92]. 


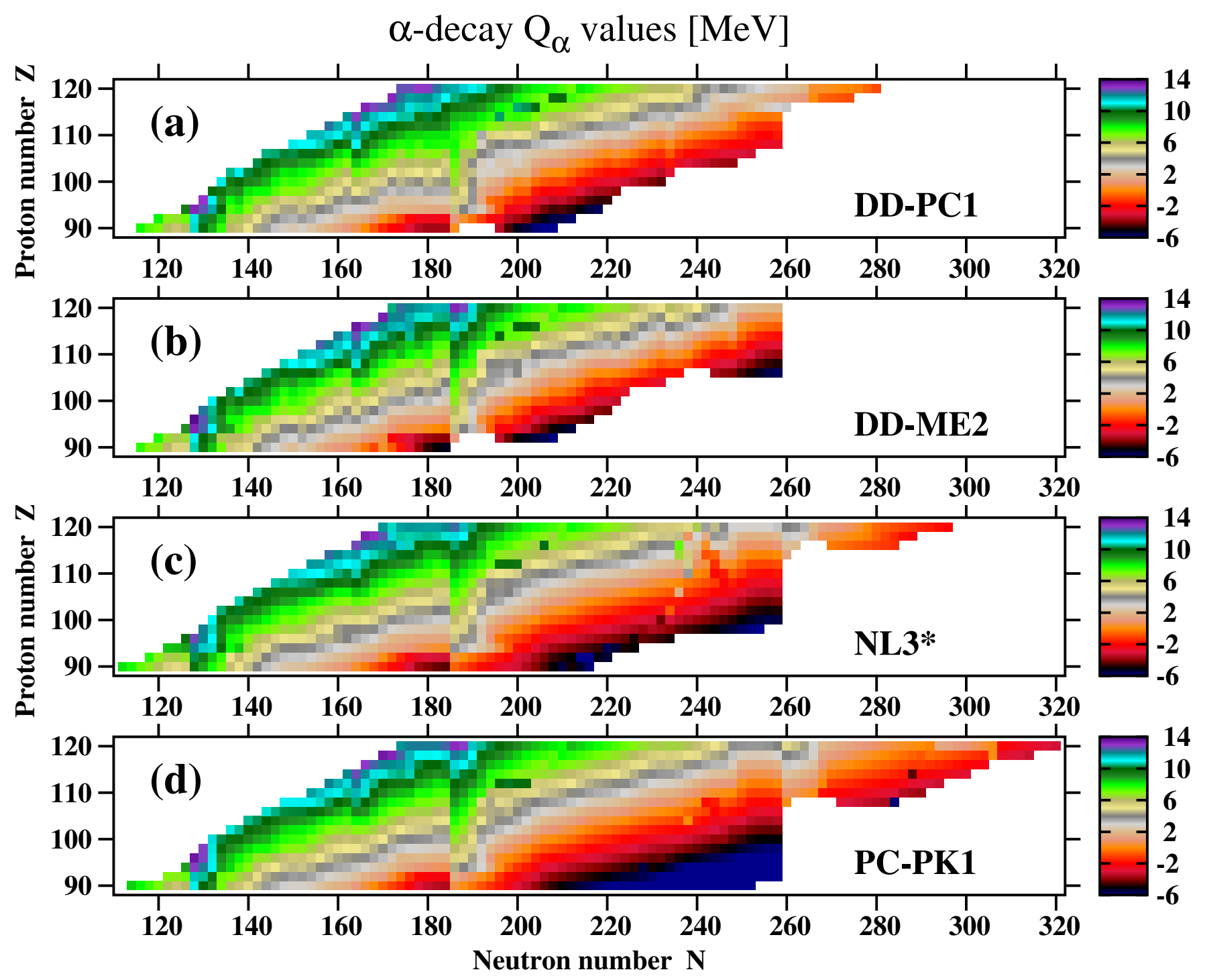

FIG. 11. The $Q_{\alpha}$ values for even-even actinides and superheavy nuclei calculated with indicated CEDFs.

(see discussion in Ref. [90]), are located at lower energies in the PC-PK1 functional as compared with the NL3* one. These features lead to the shift of the two-neutron drip line to substantially higher neutron numbers in the PC-PK1 CEDF as compared with NL3*. The sizes of the $N=258$ shell gaps are smaller by $\approx 10 \%$ and $20 \%$ in the DD-ME2 and DD-PC1 functionals as compared with the ones in the PC-PK1 and NL3*. In addition, above mentioned high- $j$ intruder orbitals in the calculations with the DD-ME2 and DD-PC1 functionals are located at higher energies as compared with the ones in NL3*. As a consequence, their two-neutron drip lines are located at lower neutron numbers as compared with the NL3*. These features are clearly seen in Fig. 5.

Fig. 8 shows the map of theoretical uncertainties $\Delta E(Z, N)$ in binding energies. These uncertainties increase drastically when approaching the neutron-drip line and in some nuclei they reach $50 \mathrm{MeV}$. Poorly defined isovector properties of CEDFs is the major reason for that (see Ref. [39]). Note that the $\Delta E(Z, N)$ spreads for the NL3*, DD-PC1 and DD-ME2 functionals are relatively modest [see Fig. $8(\mathrm{~b})]^{6}$ and the major contribution to $\Delta E(Z, N)$ is coming from the PC-PK1 functional (compare panels (a) and (b) in Fig. 8). The fact that isovector properties of the PC-PK1 functional are significanly different from those of NL3*, DD-PC1, DD-ME2 and DD-ME $\delta$ is also confirmed by the analysis of binding energies in the $\mathrm{Yb}(Z=70)$ isotopic chain (see Fig. 3 in Ref. [49]). As follows from the analysis of parametric correlations in different classes of CEDFs performed in Ref. [58], a possible reason for that could be related to overparametrization of the isoscalar channel in this class of

\footnotetext{
6 The addition of the DD-ME $\delta$ functional to this set of three functionals is not expected to modify significantly $\Delta E(Z, N)$ (see Fig. 9 in Ref. [39]).
} 
$\mathrm{CEDFs}^{7}$. This, in turn, may lead to a somewhat wrong balance of the isoscalar and isovector channels in known nuclei which reveals itself in a more pronounced way via different (as compared with other functionals) isovector dependence of binding energies in neutron-rich one.

Figure 9 presents the summary of two-neutron separation energies $S_{2 n}(Z, N)$ obtained with four employed CEDFs. Note that some discontinuities in smooth trends of the $S_{2 n}(Z, N)$ distributions as a function of neutron number are either due to the presence of substantial spherical shell gaps at $N=184$ or $N=258$ or due to the crossing of the boundaries between prolate and oblate shapes. For example, the impact of the $N=184$ spherical shell gap on the $S_{2 n}(Z, N)$ distributions is clearly visible in Figs. 9(b), (c) and (d) (see also Figs. 5 (b), (c) and (d) for deformation distributions). On the contrary, its impact is substantially suppressed in superheavy nuclei in the calculations with CEDF DD-PC1 [see Fig. 9(a)] because of the reduced role of the $N=184$ spherical shell gap in this functional.

Finally, the spreads $\Delta S_{2 n}(Z, N)$ in two-neutron separation energies are presented in Fig. 10. They are the lowest in known nuclei but in general increase with increasing neutron number. The $\Delta S_{2 n}(Z, N)$ values are quite large $\left(\Delta S_{2 n}(Z, N) \approx 2.2 \mathrm{MeV}\right)$ in the vicinity of twoneutron drip lines and the $N=184$ and $N=258$ spherical shell gaps. However, they become extremely large $\left(\Delta S_{2 n}(Z, N) \approx 4.0 \mathrm{MeV}\right)$ at the boundaries between prolate and oblate shapes. Similar to the spreads in binding energies (see discussion of Fig. 8 above), the largest contribution to the $\Delta S_{2 n}(Z, N)$ values comes from the CEDF PC-PK1. If the PC-PK1 functional is excluded from consideration these values on average decrease by a factor of 2 (compare panels (b) and (a) in Fig. 10). It is interesting that in neutron-rich deformed $N \approx 190-236$ region the $\Delta S_{2 n}(Z, N)$ values are on average comparable with those in known nuclei (see Fig. 10(b). However, they still show increased magnitudes at above discussed locations of nuclear chart.

\section{IV. $\alpha$-DECAY PROPERTIES}

In actinides and superheavy nuclei spontaneous fission and $\alpha$ emission compete and the shortest half-life determines the dominant decay channel and the total half-life. Only in the cases where the spontaneous fission half-life is longer than the half-life of $\alpha$ emission can superheavy nuclei be observed in experiment. In addition, only nuclei with half-lives longer than $\tau=10 \mu$ s are observed in experiments.

The $\alpha$ decay half-live depends on the $Q_{\alpha}$ values which

\footnotetext{
7 The analysis of Ref. [58] suggests that the number of parameters in the isoscalar channel of PC-PK1 CEDF can be reduced from 4 to 1 .
}

are calculated according to

$$
Q_{\alpha}=E(Z, N)-E(Z-2, N-2)-E(2,2)
$$

with $E(2,2)=-28.295674 \mathrm{MeV}$ [94] and $Z$ and $N$ representing the parent nucleus.

To estimate theoretical uncertainties in the predictions of the $\alpha$-decay half-lives, they were calculated using three phenomenological expressions, namely,

- the Viola-Seaborg semiempirical formula [95]

$$
\log _{10} \tau_{\alpha}=\frac{a Z+b}{\sqrt{Q_{\alpha}}}+c Z+d
$$

employing two sets of parametrizations. The first one with the parameters $a=1.66175, b=-8.5166$, $c=-0.20228$ and $d=-33.9069$ has been fitted in Ref. [96]. This set and the results obtained with it are labelled further as VSS-1989. Another set has been defined in Ref. [97] and its paramaters are: $a=1.64062, b=-8.54399, c=-0.19430$ and $d=-33.9054$. The label VSS-2005 is used for it and its results.

- phenomenological first modified Brown fit (mB1) [98]

$$
\log _{10} \tau_{\alpha}=\frac{a(Z-2)^{b}}{\sqrt{Q_{\alpha}}}+c
$$

with the parameters $a=13.0705, b=0.5182$, $c=-47.8867$. This set and its results are labeled further as MB-2016.

- phenomenological Royer model [99]

$$
\log _{10} \tau_{\alpha}=\frac{a Z}{\sqrt{Q_{\alpha}}}+b A^{\frac{1}{6}} \sqrt{Z}+c
$$

with the parameters $a=1.5864, b=-1.1629$ and $c=-25.31$ of Ref. [99]. Its results are labeled further as Royer-2000.

These phenomenological expressions employ different functional dependencies (in particular, they show different dependencies on proton and mass numbers) and are fitted to different sets of experimental data. This is expected to lead to different predictions for $\tau_{\alpha}$ in high- $Z$ and neutron-rich nuclei.

The $Q_{\alpha}$ values calculated with the DD-PC1, DD-ME2, NL3* and PC-PK1 functionals are presented in Fig. 11. One can see that for a fixed value of $Z$ with increasing neutron number the $Q_{\alpha}$ values in general decrease. They are positive in proton-rich nuclei as well as in the nuclei located close to the $\beta$-stability line. The $Q_{\alpha}$ values experience a substantial increase at shell closure with $N=184^{8}$ (see Fig. 11 in the present paper as well as Fig.

\footnotetext{
${ }^{8}$ Similar increase is also seen in the vicinity of the $N=258$ shell closure in the calculations with the NL3* and PC-PK1 CEDFs [see Fig. 11(c) and (d)].
} 


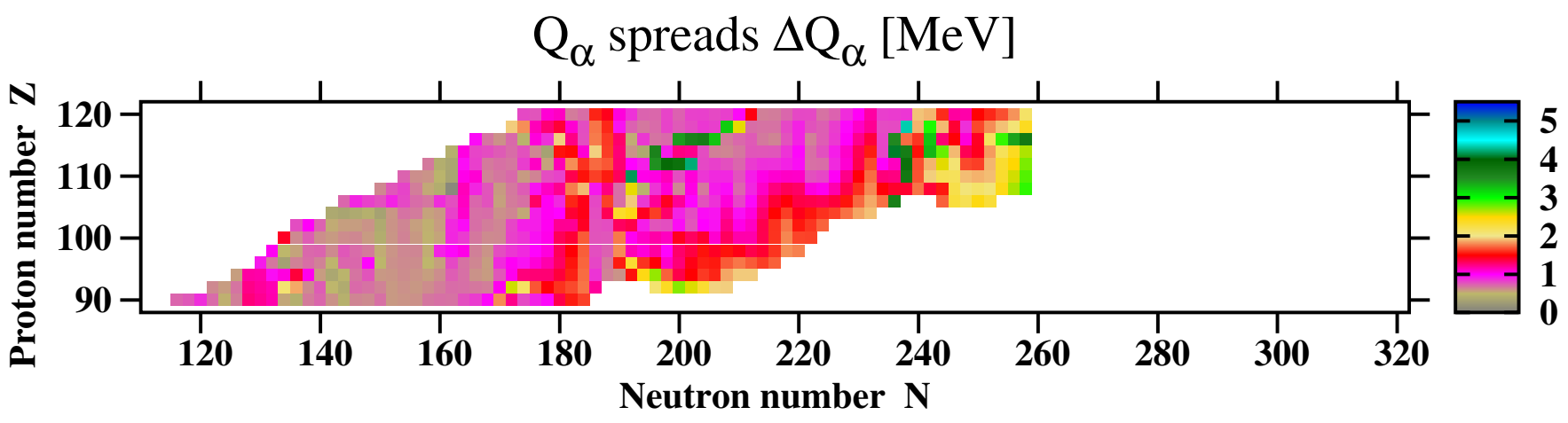

FIG. 12. The $Q_{\alpha}$ spreads $\Delta Q_{\alpha}(Z, N)$ as a function of proton and neutron number. $\Delta Q_{\alpha}(Z, N)=\left|Q_{\alpha}^{\max }(Z, N)-Q_{\alpha}^{\min }(Z, N)\right|$, where $Q_{\alpha}^{\max }(Z, N)$ and $Q_{\alpha}^{\min }(Z, N)$ are the largest and smallest $Q_{\alpha}$ values obtained with four employed CEDFs for the $(Z, N)$ nucleus.

14 in Ref. [59]); note that the effect of this shell closure is washed out in the $Z>110$ nuclei for the DD-PC1 functional. With subsequent increase of neutron number the $Q_{\alpha}$ values become first smaller, then they become close (or equal) to zero and with further increase of $N$ they get more and more negative. Note that $\alpha$-decay is energetically not possible for $Q_{\alpha} \leq 0 \mathrm{MeV}$. Thus, very neutron-rich nuclei cannot decay by $\alpha$-emission.

Note that general trends in the development of the $Q_{\alpha}$ values as a function of proton and neutron number are similar in all functionals. The major differences are related to the location of the two-neutron drip line, the impact of the $N=184$ and $N=258$ shell closures and the location of the boundaries between prolate and oblate nuclear shapes. These differences between the functionals are summarized in Fig. 12 which shows the $Q_{\alpha}$ spreads $\Delta Q_{\alpha}(Z, N)$ as a function of proton and neutron number. The largest spread in the predictions exists in the island centered around $Z \sim 110, N \sim 198$ in which $\Delta Q_{\alpha}(Z, N)>3 \mathrm{MeV}$. This spread emerges from different predictions of the boundaries in the $(Z, N)$ plane between prolate and oblate shapes (see Fig. 5) and coincides with the largest spread in calculated ground state deformations (see Fig. 7). The next region with the largest differences in the predictions is located between neutron numbers $\mathrm{N}=236$ and $\mathrm{N}=258$ (see Fig. 12). However, these differences are not critical because (i) this region is not expected to play a role in the r-process, (b) expected $\alpha$ decay half-lives exceed $10^{20} \mathrm{~s}$ (see Fig. 13) and (c) many of the nuclei in this region are not expected to decay by $\alpha$-emission. High $\Delta Q_{\alpha}$ values $\left(\Delta Q_{\alpha}(Z, N) \approx 1.5 \mathrm{MeV}\right)$ are observed near shell closure at $N=184$ and in very neutron-rich nuclei near two-neutron drip line. This is a consequence of the difference in the predictions of the ground state properties such as deformations in the nuclei near $N=184$ (see Ref. [59]) and general deterioration of predictive power of nuclear models on approaching neutron drip line (see Ref. [39]). In other regions of nuclear chart, $\Delta Q_{\alpha}(Z, N) \leq 1.0 \mathrm{MeV}$ with smallest spreads seen in the $N<180$ nuclei.

Note that the inclusion of dynamical correlations (for example, by means of 5 dimensional collective Hamiltonian) can locally modify the binding energies and $Q_{\alpha}$ values $[63,68,86]$ but they have the largest impact on transitional nuclei which represent only minor part of the nuclei under study. For well deformed nuclei, the impact of dynamical correlations on $Q_{\alpha}$ values is rather modest [63]. Thus, their inclusion is not expected to change drastically global picture for the behavior of $Q_{\alpha}$.

Calculated $\alpha$-decay half-lives $\tau_{\alpha}$ (in logarithmic scale) obtained with the VSS-2005 empirical formula for four CEDFs are shown in Fig. 13. Other phenomenological formulas such as VSS-1989, MB-2016 and Royer-2000 give similar results; thus, they are not shown. For a given isotope chain the calculated half-lives generally increase with increasing neutron number. This trend is interrupted only at the $N=184$ and $N=258$ shell closures. The consequence of this feature is the fact that traditional experimental technique of detecting superheavy nuclei by $\alpha$-decay will not work in neutron-rich nuclei because they can decay faster by spontaneous fission. Note that $\alpha$-decay is energetically forbidden for a large group of very neutron-rich nuclei located in the vicinity of neutron-drip line. In such nuclei as well as in those which have very large $\tau_{\alpha}$ values, the competition of spontaneous fission, neutron induced fission, $\beta$-decay, and neutron emission will define the leading channel of decay in the r-process calculations.

It is important to remember that the typical timescale of the r-process is in the seconds range [2-4]. Fig. 13 clearly illustrates that with few exceptions the nuclei located to the right of white lines have $\alpha$-decay half-lives exceeding $10 \mathrm{~s}$. Thus, $\alpha$-decay half-lives of these nuclei are longer than the typical timescale of the r-process and, as a consequence, their alpha-decays will not affect the r-process simulations. These white lines in Fig. 13 also outline the region of nuclear chart in which traditional experimental measurements of superheavy nuclei based on the $\alpha$-decays are possible: these are the regions located near and to the left of these white lines.

Theoretical uncertainties in the predictions of $\alpha$-decay half-lives given via the $\Delta\left(\log _{10}\left(\tau_{\alpha}\right)\right)$ spreads are summa- 


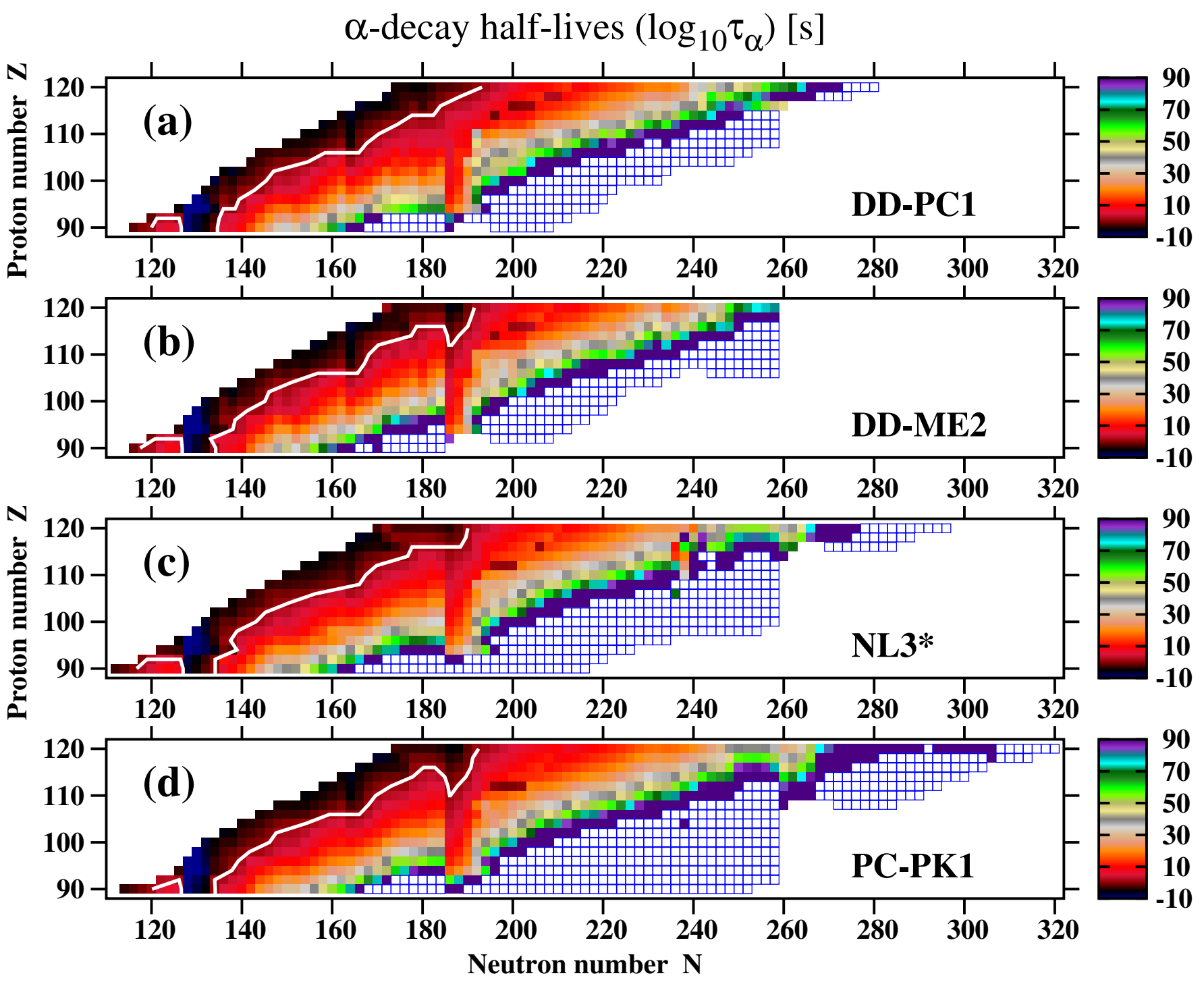

FIG. 13. Calculated $\log _{10}\left(\tau_{\alpha}\right)$ values of the $\alpha$-decays for even-even superheavy nuclei obtained with the VSS-2005 version of Viola-Seaborg semi-empirical formula for four indicated CEDFs. Open squares are used for the nuclei in which $\alpha$-decay is energetically forbidden. The white line corresponding to $\log _{10}\left(\tau_{\alpha}\right)=1.0$ outlines the region of nuclei in which the alpha-decay half-live is smaller than $10 \mathrm{~s}$.

rized in Fig. 14. The comparison of the panels (a) and (b) clearly shows that these uncertainties mostly emerge from the differences in the predictions of the $Q_{\alpha}$ values by different functionals. These uncertainties exceed 50 orders of magnitude in the nuclei located in the vicinity of two-neutron drip line and in some nuclei around $Z \approx 108, N \approx 198$ (see Fig. 14). The uncertainties in $\tau_{\alpha}$ originating from different empirical formalas [see Eqs. (19), (20) and (21)] are significantly smaller [see Fig. 14(b)]. For absolute majority of the nuclei they are smaller than 5 orders of magnitude and for proton-rich nuclei and the nuclei located close to the beta-stability they are very small. They are larger than 10 orders of magnitude only in neutron-rich nuclei located in close vicinity of two-neutron drip line. Combined theoretical uncertainties in $\tau_{\alpha}$ emerging from the use of four empir- ical formulas and four CEDFs are summarized in Fig. 14(c). One can see that for almost half of nuclei they exceed 10 orders of magnitude; these nuclei are located on neutron-rich side of nuclear chart. However, these uncertainties are not very critical since the $\alpha$-decay lifetimes become extremely large in such nuclei (see Fig. 13) so $\alpha$ decay can compete neither with fission nor with $\beta$-decay. Note also that the removing of the PC-PK1 functional from consideration does not change appreciably theoretical uncertainties in the predictions of $\alpha$-decay half-lives (compare Figs. 14 (c) and (d)). 


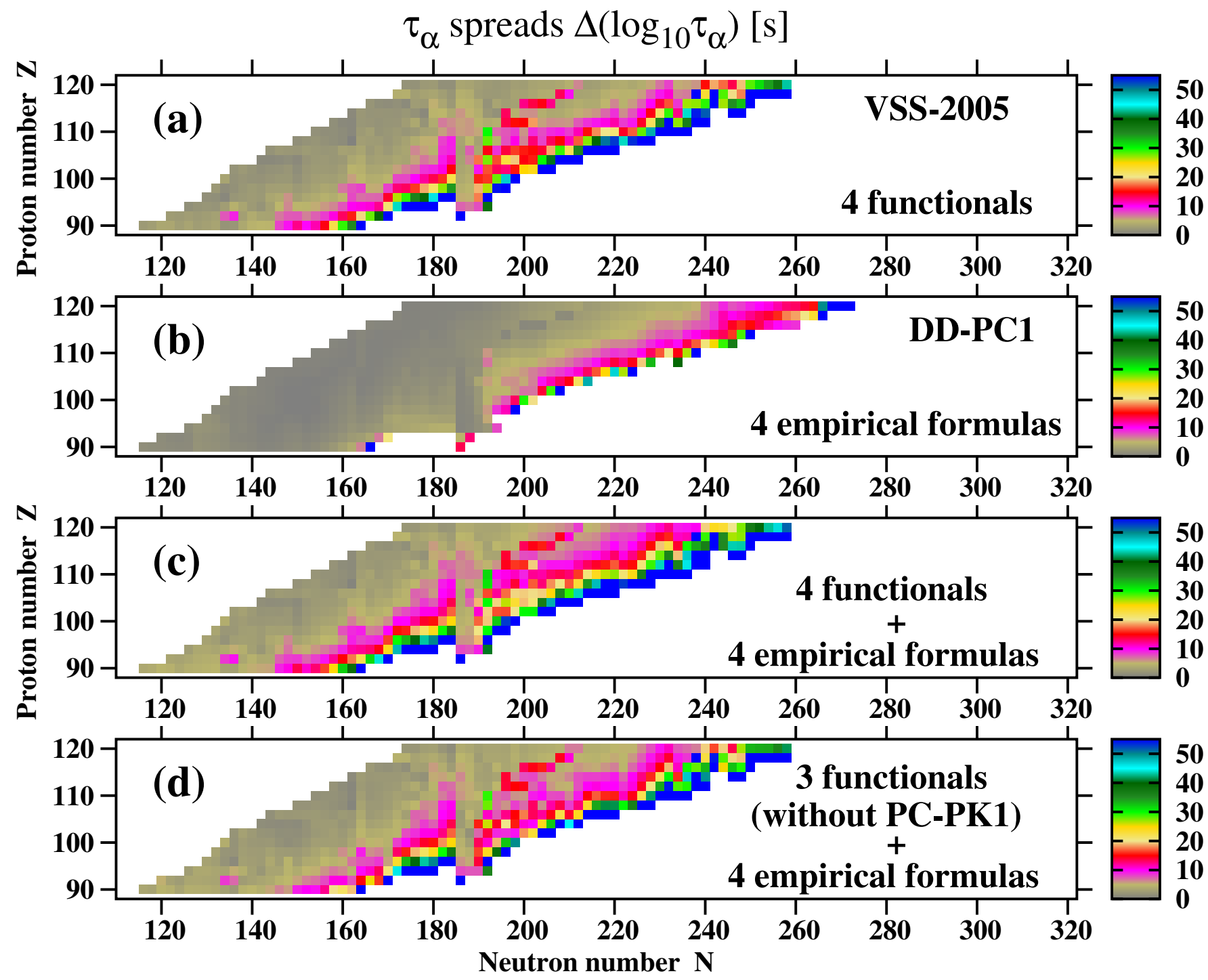

FIG. 14. The $\tau_{\alpha}$ spreads $\Delta \tau_{\alpha}(Z, N)$ as a function of proton and neutron number. $\Delta \tau_{\alpha}(Z, N)=\left|\tau_{\alpha}^{\max }(Z, N)-\tau_{\alpha}^{\min }(Z, N)\right|$, where $\tau_{\alpha}^{\max }(Z, N)$ and $\tau_{\alpha}^{\min }(Z, N)$ are the largest and smallest $\tau_{\alpha}$ values obtained with selected set of functionals and empirical formulas. Panel (a) shows these spreads obtained with four employed CEDFs and VSS-2005 empirical formula. DD-PC1 CEDF and four empirical formulas are used in panel (b). Panel (c) presents combined spread of $\tau_{\alpha}$ obtained with four CEDFs and four empirical formulas. Panel (d) is the subversion of panel (c) in which the PC-PK1 functional is excluded.

\section{FISSION PROPERTIES}

A. Primary fission barriers

The distributions of primary fission barriers ${ }^{9}$ (PFB) heights in the $(Z, N)$ plane obtained with employed functionals are shown in Fig. 15. Fig. 16 presents the maps of the nuclei in the region under study in which outer fission barriers are higher than inner ones in the RS-RHB

\footnotetext{
9 The highest in energy fission barrier (among inner and outer ones) is called primary and it plays an important role in the r-process modeling (see Ref. [85]).
}

calculations (see Figs. 3 and 4 for more details). It also illustrates that the importance of outer fission barriers in stabilization of nuclei in general decreases on going from light actinides to superheavy nuclei because of increased importance of Coulomb interaction (compare Figs. 4 and 3). Fig. 16 also demonstrates the impact of octupole deformation (as obtained in RA-RHB calculations) on the outer fission barriers and on their heights with respect of inner ones. The lowering of outer fission barrier due to octupole deformation indicates that asymmetric fission becomes dominant, while the absence of the impact of octupole deformation on outer fission barrier height tells that fission will be symmetric.

Fig. 16 shows that similar regions in the $(Z, N)$ plane, in which the outer fission barriers are higher in energy 


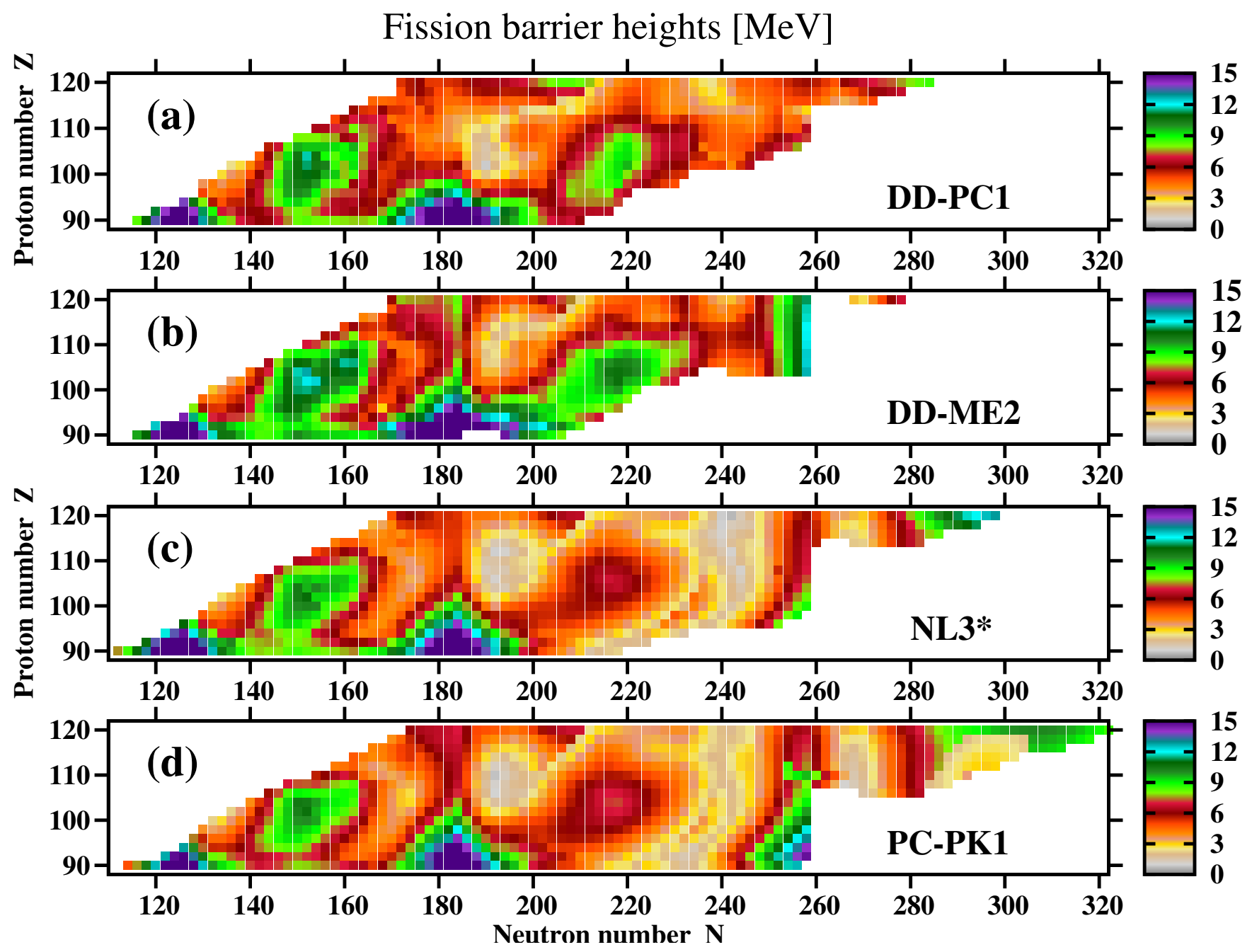

FIG. 15. The heights of primary fission barriers (in MeV) obtained in axial RS-RHB and RA-RHB calculations as a function of proton and neutron numbers for nuclei located between two-proton and two-neutron drip lines.

than inner ones in the RS-RHB calculations, appear in the calculations with all employed functionals. However, these regions are substantially larger in the density dependent functionals (such as DD-PC1 and DD-ME2) as compared with CEDFs NL3* and PC-PK1. Octupole deformation does not affect outer fission barriers in the nuclei located in the $Z \sim 110, N \sim 240$ region, in the $N \leq 120$ nuclei as well as in the nuclei located not so far away from $N \approx 180$. On going away from the latter two regions, octupole deformation first starts to reduce the heights of outer fission barriers but they still remain higher in energy than inner ones. Further transition away from these regions leads to the reduction of the heights of outer fission barriers below the inner ones due to the impact of octupole deformation.

Fig. 15 reveals a lot of similarities in the predictions of the global structure of the maps of fission barrier heights obtained with employed functionals. The highest PFBs are predicted in the islands of low- $Z$ nuclei centered around spherical shell closures with $N=126$ and
$N=184$ (and $N=258$ in the case of the PC-PK1 functional). Fission barriers reach $15 \mathrm{MeV}$ in the centers of these islands. Next island with high fission barriers exists around $Z \approx 100, N \approx 150$. Left bottom part of this island coincides with the region of actinides (see, for example, Fig. 7 in Ref. [62]) in which the heights of fission barriers have been experimentally measured. Relativistic mean field calculations with the NL3*, PC-PK1 and DD-PC1 functionals performed by different groups rather well describe inner and outer fission barriers in actinides [62-64, 69]. Note also that the spreads of the heights of inner fission barriers obtained with these three functionals in the $Z \approx 100, N \approx 150$ island are relatively small (approximately $1 \mathrm{MeV}$ or less) for the majority of nuclei in this island (see Fig. 3b in Ref. [47]). On the contrary, the DD-ME2 functional predicts somewhat higher fission barriers in this island (see Fig. 15b) which leads to somewhat higher spreads $\Delta E^{B}$ in the heights of primary fission barriers (see Fig. 17a). The island of low fission barriers is seen near $Z \approx 108, N \approx 192$ in all function- 


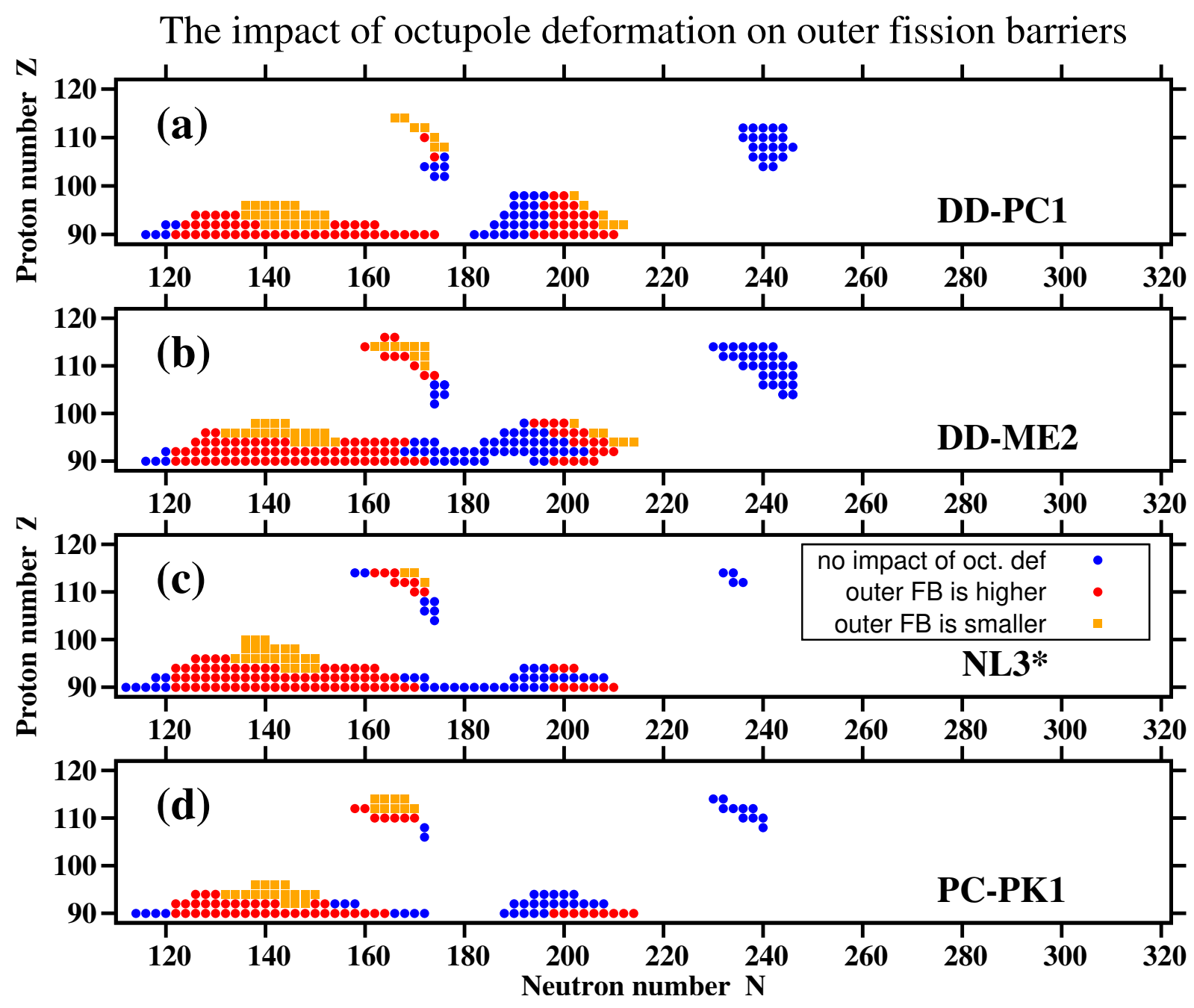

FIG. 16. The distributions of the nuclei, in which the outer fission barrier is higher than inner one in the RS-RHB calculations, in the $(Z, N)$ plane for four employed CEDFs. Different types of symbols are used to indicate the impact of octupole deformation on outer fission barriers of these nuclei. Blue solid circles are used for the nuclei the heights of outer fission barriers of which are not affected by the inclusion of octupole deformation. Solid red circles/orange squares are used for the nuclei in which outer fission barrier is affected by octupole deformation in the RA-RHB calculations and is higher/lower than inner fission barrier.

als. Then another island of high fission barriers centered around $Z \approx 104, N \approx 216$ is formed. The highest fission barriers reaching $10-11 \mathrm{MeV}$ in the center of this island are predicted by the DD-ME2 functional (see Fig. 15b). Somewhat lower fission barriers (with approximately 9 $\mathrm{MeV}$ height in the center of the island) are predicted by the DD-PC1 functional (see Fig. 15a). Fission barriers with height of approximately $6 \mathrm{MeV}$ appear in broad region of this island in the calculations with the NL3* and PC-PK1 CEDFs (see Figs. 15c and d).

With increasing neutron number the predictions start to differ substantially. The NL3* and PC-PK1 functionals predict extremely low fission barriers with heights of around $2 \mathrm{MeV}$ or less for the band of nuclei around $N \approx 240$ (see Figs. 15c and d). No such band is formed in the calculations with DD-ME2 and DD-PC1 functionals (see Figs. 15a and b). This could have a drastic impact on the creation of superheavy elements in the r-process because the nuclear flow during most of neutron irradiation step of the r-process follows the neutron drip line and produces in tens of $\mathrm{ms}$ the heaviest drip line nuclei (see the discussion in Sec. 4 of Ref. [12]). However, this nuclear flow will most likely be terminated at $N \approx 240$ nuclei in the calculations with NL3* and PC-PK1 since fission will be much faster than neutron capture. Thus, no superheavy nuclei are expected to be formed in the r-process calculations based on fission barriers obtained with these two functionals. This is similar to the results of the r-process simulations based on nonrelativistic models (such as Skyrme DFT with HFB-14 functional, Thomas-Fermi (TF) model and Finite Range Droplet Model (FRDM)) with low fission barriers in the vicinity of neutron drip line $[12,15]$. On the contrary, the formation of superheavy elements in the r-process is more likely in the calculations based on the DD-ME2 and DD-PC1 functionals since the $(Z, N)$ region near neutron 


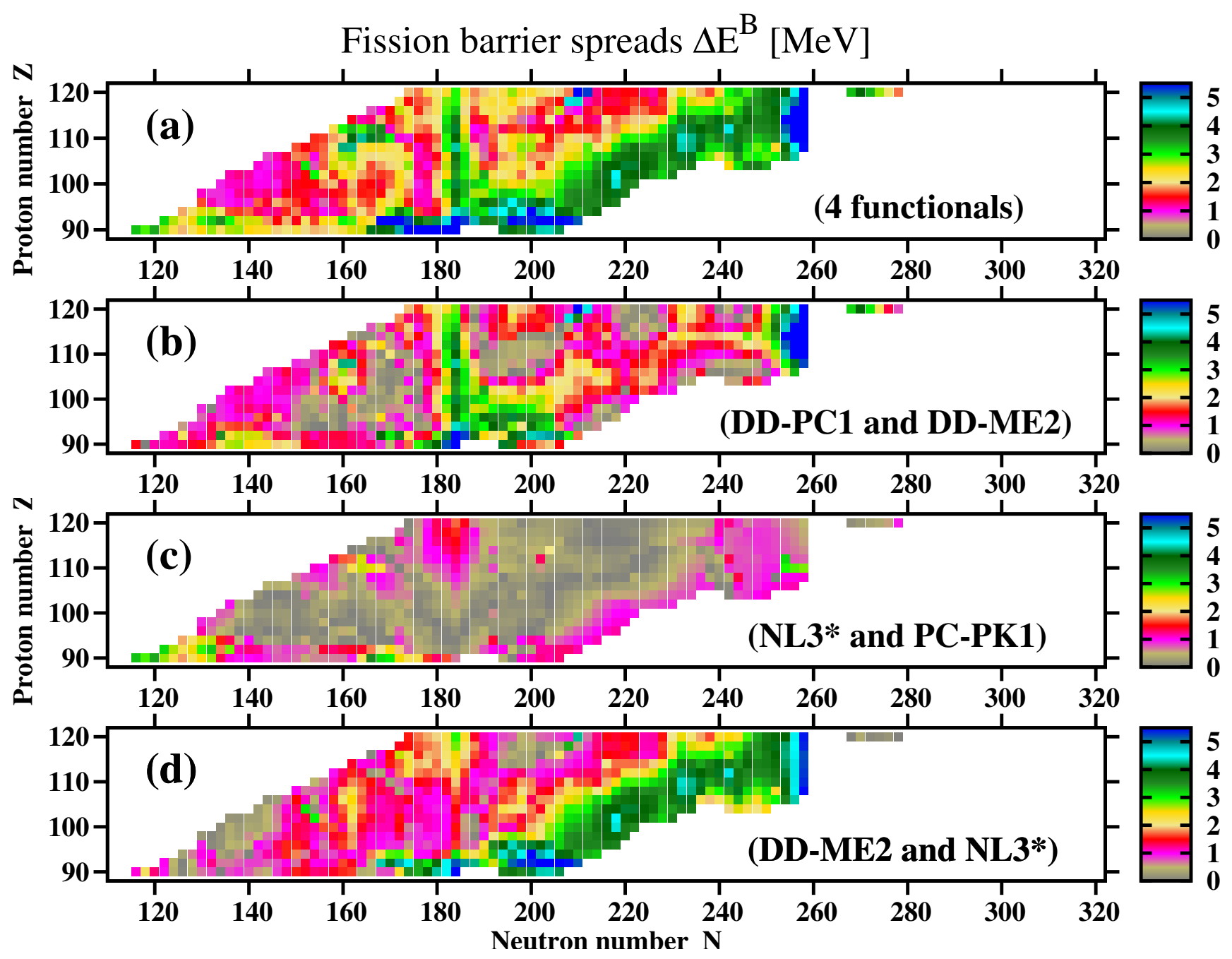

FIG. 17. (a) The spreads $\Delta E^{B}$ of the heights of primary fission barriers as a function of proton and neutron numbers. $\Delta E^{B}(Z, N)=\left|E_{\max }^{B}(Z, N)-E_{\min }^{B}(Z, N)\right|$, where, for given $Z$ and $N$ values, $E_{\max }^{B}(Z, N)$ and $E_{\min }^{B}(Z, N)$ are the largest and smallest heights of inner fission barriers obtained with employed set of four functionals. (b-d) The spreads $\Delta E^{B}$ obtained for indicated pairs of the functionals.

drip line is characterized by relatively high fission barriers (see Figs. 15a and b). As a consequence, neutron capture is expected to proceed faster than fission and nuclear flow during neutron irradiation step of the r-process could extend to higher proton numbers $[12,15]$. To a degree this is similar to the r-process simulations based on the models (such as Extended Thomas Fermi (ETFSI) model) with high fission barriers near neutron drip line (see Ref. [15]).

Further increase of neutron number leads to a rise of the heights of primary fission barriers (PFBs) and the formation of the band of nuclei near shell closure at $N=258$ with the heights of PFBs exceeding $6 \mathrm{MeV}$ (see Fig. 15b, $\mathrm{c}$ and $\mathrm{d}$ ). In some nuclei they even reach $12-15 \mathrm{MeV}$ in the calculations with PC-PK1 and NL3* CEDFs (see Fig. $15 \mathrm{c}$ and $\mathrm{d}$ ). Note that this process is suppressed in the calculations with CEDF DD-PC1 (see Fig. 15a) because of reduced impact of the $N=258$ spherical shell gap on the ground state deformations (see Fig. 5a). Nuclear landscape extends substantially beyond $N=258$ in the calculations with NL3* and PC-PK1 (see Fig. 15c and d). In this region we again see the alteration of the regions of low (near $N \approx 268$ ) and high (near $N \approx 280$ and above) PFBs. Note that in the $N>258$ region toroidal shapes $[65,100]$ could become the lowest in energy solutions. This has been verified in the calculations with DD-PC1 functional in Ref. [66] but has not been checked for other functionals.

B. Theoretical uncertainties in primary fission barriers and their sources

The spreads $\Delta E^{B}$ of the heights of primary fission barriers as a function of proton and neutron numbers for four employed functionals are shown in Fig. 17(a). 
One can see that on the average they are moderate (on the level of $1.0-1.5 \mathrm{MeV}$ ) in the neutron poor region of actinides centered around $(Z \sim 100, N \sim 140)$. Then the spreads start to increase with a small island of high $\Delta E^{B} \approx 4.0 \mathrm{MeV}$ values seen in superheavy nuclei around $Z \approx 110, N \approx 164$. Further increase of neutron number leads to the band of high $\Delta E^{B} \approx 4.0 \mathrm{MeV}$ values for the nuclei with $N \approx 184$. The sources of these uncertainties are directly related to the differences in the predictions of the ground state properties of these nuclei: spherical ground states are predicted in these nuclei in the calculations with PCPK1, DD-ME2 and NL3* functionals [see Figs. 5(b),(c) and (d)] while the DD-PC1 functional prefers oblate ground states in superheavy $N \approx 184 \mathrm{nu}-$ clei (see Fig. 5(a)). After crossing this band, theoretical uncertainties in the heights of primary fission barriers substantially decrease and for the majority of the nuclei located inside the triangle with the sides $N=188$ [for $Z=96-120$ ], $Z=120$ [for $N=188-240$ ] and $[Z=96, N=188$ to $Z=120, N=240]$ they are in general better than $2.5 \mathrm{MeV}$ and in many nuclei they are even better than $1.5 \mathrm{MeV}$. However, the $\Delta E^{B}$ spreads start to increase again on approaching two-neutron drip line. Here they form quite wide band of the nuclei parallel to two-neutron drip line in which $\Delta E^{B}$ is close to 4.0 MeV. Even higher spreads reaching $5.5 \mathrm{MeV}$ are seen near shell closure at $N=258$.

The analysis of the spreads $\Delta E^{B}$ allows to identify major sources of theoretical uncertainties in the predictions of the heights of PFBs. These could be reduces to two major contributors, namely, underlying singleparticle structure mostly affecting the ground state properties and nuclear matter properties of employed CEDFs. To facilitate the discussion we will consider the $\Delta E^{B}$ spreads for the pairs of selected functionals.

The lowest spreads exist for the pair of the NL3* and PC-PK1 functionals (see Fig. 17c): $\Delta E^{B} \leq 0.5 \mathrm{MeV}$ for absolute majority of the nuclei and only in specific regions of nuclear chart it is higher. Even in those regions it is higher than $1 \mathrm{MeV}$ only for limited set of the nuclei. These regions are: (i) the actinides around $N=126$, (ii) the $Z=90$ and 92 actinides with $N \approx 170$, (iii) superheavy nuclei in the vicinity of the $Z=120$ and $N=184$ lines, (iv) very neutron-rich nuclei in the vicinity of two-neutron drip line and (v) the band of the nuclei around $N \approx 246$. The differences in the predictions of the heights of outer fission barriers are responsible for the spreads in the region (i). At present, their source is not clear. The spreads $\Delta E^{B}$ seen in the region (ii) are due both to different proton and neutron dependencies of the impact of octupole deformation on outer fission barriers in the NL3* and PC-PK1 functionals (compare panels (c) and (d) of Fig. 16) and the fact that in some nuclei we compare the heights of outer and inner fission barriers. In the region (iii), the large $\Delta E^{B}$ values are due to the differences in the predictions of the spherical shell closures at $Z=120$ and $N=184$ and the densities of the single-particle states in their vicinities (see Fig. 1 in Ref. [59] and the discussion in this reference). Slightly different isovector properties of the NL3* and PC-PK1 functionals (see Table II) may be responsible for the divergence of their predictions in the region (iv). The large $\Delta E^{B}$ values in the region (v) are due to prolate-oblatespherical shape coexistence which takes place in slightly different regions of the $(Z, N)$ chart in these two functionals [compare Figs. 5 (c) and (d)].

The comparison of the predictions of the NL3* and PC-PK1 functionals for the fission barriers is quite illuminating since it shows in a global way a number of important features. First, apart of the regions (i)-(v) the comparable (typically within $0.5 \mathrm{MeV}$ ) predictions for the heights of PFBs are obtained on a global scale by these two functionals despite the fact that they differ substantially in the predictions of the ground states energies in neutron-rich nuclei (see the discussion of Fig. 8). Thus, the description of the ground state energies is to a degree decoupled from the description of fission barriers; the latter depends on the relative energies of the saddle and the ground state. As a consequence, good description of the ground state energies does not guarantee good description of the fission barriers and vice versa. Second, the differences in the predictions of the $\mathrm{PFB}$ heights seen in the regions (iii) and (v) are related to the differences in the predictions of the ground states properties, which in turn are defined by the differences in the underlying single-particle structure. Third, if to exclude the regions (i)-(v) from consideration it becomes clear that some differences in nuclear matter properties such as the symmetry energy $J$ and its slope $L_{0}$ (see Table II) do not lead to important differences in the predictions for PFBs. Fourth, comparable global predictions for the $\mathrm{PFBs}$ are obtained despite underlying differences in the basic structure of the functionals and their fitting protocols. The NL3* functional includes meson exchange of finite range, while $\mathrm{PC}-\mathrm{PK} 1 \mathrm{CEDF}$ does not have mesons and thus it has zero range interactions (see Ref. [58]). The fitting protocol of the CEDF NL3* is based on 12 spherical nuclei and includes empirical data on nuclear matter properties (see Ref. [43]), while the one for PCPK1 includes only data on binding energies (60 spherical nuclei) and charge radii (17 spherical nuclei) [50]. Note that the NL3* and PC-PK1 functionals have 6 and 9 parameters, respectively. However, the analysis of parametric correlations shows that in reality there are only 5 and 6 independent parameters in these two functionals $[46,58]$.

Next we consider the spreads $\Delta E^{B}$ obtained with the NL3*/DD-ME2 pair of the functionals (see Fig. 17d). These two functionals have almost identical fitting protocols (see Refs. [42, 43]). The only difference is the fact that DD-ME2 fitting protocol uses 3 experimental data points on neutron skins as compared with 4 in NL3* but the impact of this difference is expected to be small. Thus, larger values of the $\Delta E^{B}$ spreads in the NL3*/DDME2 pair as compared with the ones in the NL3*/PC$\mathrm{PK} 1$ pair are related to the basic difference of these two 
functionals, namely, to the implementation of density dependence. The DD-ME2 functional has explicit dependence of the meson-nucleon coupling on the nucleonic density, while NL3* employs cubic and quartic powers of the $\sigma$ meson for density dependence (see Sect. II of Ref. [39] for details). In addition, the nuclear matter properties (in particular, the symmetry energy $J$ and its slope $L_{0}$ ) of these two functionals differ substantially (see Table II) and this difference is expected to contribute into the increase of the spreads $\Delta E^{B}$ obtained for the NL3*/DDME2 pair as compared with those for the NL3*/PC-PK1 pair.

Finally, the $\Delta E^{B}$ spreads for the DD-PC1/DD-ME2 pair of the functionals are presented in Fig. 17b. Nuclear matter properties of these two functionals are close to each other and they are located within the limits of the SET2b constraint set on the experimental/empirical ranges for the quantities of interest derived in Ref. [51] (see Table II). However, fitting protocols of these two functionals are drastically different: CEDF DD-ME2 is fitted to the properties of 12 spherical nuclei (see Ref. [43]) while DD-PC1 is defined by the properties of 64 deformed rare-earth nuclei and actinides (see Ref. [44]). As a result of this difference in fitting protocols, the largest $\Delta E^{B}$ spreads appear in the vicinity of spherical shell closures at $N=184$ (with $\Delta E^{B}$ reaching $4.0 \mathrm{MeV}$ ) and $N=258$ (with $\Delta E^{B}$ reaching $5.5 \mathrm{MeV}$ ) [see Fig. 17b]. Indeed, the impact of these shell closures on the equilibrium deformation differs substantially in these two functionals (compare Figs. 5(a) and (b) and see Fig. 7(b)) and this is a reason for increased $\Delta E^{B}$ spreads.

\section{The comparison with the results obtained in non-relativistic calculations}

It is interesting to compare the global trends of the heights of PFB in the $(Z, N)$ plane obtained in the RHB calculations (see Fig. 15) with those obtained in earlier non-relativistic calculations for which the maps similar to those presented in Fig. 15 are available. Note that similar to our calculations all these non-relativistic calculations have been performed only for axial nuclear shapes.

Fission barriers obtained in Gogny DFT calculations with $\mathrm{D} \mathrm{M}^{*}$ functional are presented in Fig. 12 of Ref. [23]. These calculations cover the region from two-proton drip line up to the nuclei with two-neutron separation energies of $S_{2 n}=4.0 \mathrm{MeV}$. In these calculations, the fission barriers of the $N \leq 186$ nuclei typically exceed 6 $\mathrm{MeV}$ and in a number of these nuclei their heights are close to $12 \mathrm{MeV}$. Then fission barriers in the $N \approx 190-$ 210 nuclei are lower than $4 \mathrm{MeV}$ but they increase to approximately $8 \mathrm{MeV}$ on approaching $S_{2 n}=4.0 \mathrm{MeV}$ line. The differences in the predictions of the heights of PFB obtained in the CDFT and Gogny DFT calculations (compare Fig. 15 with Fig. 12 of Ref. [23]) are in part related to the differences in the predictions of ground state properties (compare Fig. 5 in the present paper with Fig. 5 in Ref. [23]).

Our results for fission barriers (Fig. 15) could also be compared with those obtained in non-relativistic DFTs with the BCPM and HFB14 functionals and FRLDM (see Fig. 7 in Ref. [18]). The calculations with HFB14 predict very low fission barriers (with $E^{B}<4 \mathrm{MeV}$ ) for the $Z \geq 110$ nuclei with exceptionally low fission barriers $\left(E^{B}<2 \mathrm{MeV}\right)$ in many nuclei located in the $N \approx 184-$ 210 region (see middle panel of Fig. 7 in Ref. [18] and Fig. 13 in Ref. [85]). The RHB calculations predict in general higher fission barriers (as compared with HFB14 ones), but similar island of low fission barriers is seen near $Z \approx 108, N \approx 192$ in all functionals (see Fig. 15). However, this island is narrower as compared with the HFB14 one. Fission barriers obtained with the BCPM functional and FRLDM are somewhat higher than those obtained with HFB14 (compare top and bottom panel with middle panel of Fig. 7 in Ref. [18]). However, they share the same general structure in the $(Z, N)$ plane.

Fission barriers calculated in the DFT framework with Skyrme SLy6, SkI3, SV-min and SV-bas functionals are presented in Fig. 5 of Ref. [21]. Unfortunately, the colormap used in this figure does not allow to extract the details in the most interesting energy range of $6-10$ $\mathrm{MeV}^{10}$. However, the region of low fission barriers (with $E^{B}<4 \mathrm{MeV}$ ) similar to that discussed above appear in all functionals for $N \approx 190-210, Z \approx 94-120$. Fission barriers obtained in the TF and ETFSI models for the $(Z=84-120, N=140-236)$ and $(Z=84-115, N=$ $140-216)$ regions of nuclear chart are presented in Fig. 2 of Ref. [15]. Both of these models show the island of low fission barriers centered around $Z \approx 110, N \approx 192$. In general, the ETFSI results are close to above mentioned results obtained with Skyrme EDFs.

\section{CONCLUSIONS}

The systematic investigation of the ground state and fission properties of even-even actinides and superheavy nuclei with $Z=90-120$ from the two-proton up to twoneutron drip lines has been performed for the first time in the framework of covariant density functional theory. Four state-of-the-art CEDFs such as DD-PC1, DD-ME2, $\mathrm{NL}^{*}$ and PC-PK1 are used in this study. They represent the major classes of the CDFT models which differ by basic assumptions and fitting protocols. This allows a proper assessment of systematic theoretical uncertainties for physical observables of interest. Obtained results provide a necessary theoretical input for the r-process modeling in heavy nuclei and, in particular, for the study of fission cycling. The main results can be summarized as follows:

10 Better colormap for the fission barrier height distribution in the $(Z, N)$ plane obtained with the Skyrme SV-min functional is used in Fig. 4 of Ref. [22]. 
- Quadrupole deformations of calculated ground states and related theoretical uncertainties have been investigated. It turns out that four employed functionals predict very similar deformations for the majority of the nuclei. However, large theoretical uncertainties in quadrupole deformation exist for some nuclei but they are well localized in the $(Z, N)$ plane. These uncertainties are mostly due to the uncertainties in the predictions of the underlying single-particle structure. They are dominated by the uncertainties in the predictions of both spherical shell closures at $N=184, N=258$ and $Z=120$ (for the $N<190$ nuclei) and deformed single-particle structures leading to somewhat different boundaries in the $(Z, N)$ plane between the regions with oblate and prolate shapes. The differences in nuclear matter properties of employed functionals have only marginal impact on theoretical uncertainties related to calculated quadrupole deformations.

- Theoretical uncertainties $\Delta E(Z, N)$ in binding energies, emerging from poorly defined isovector properties of CEDFs, increase drastically when approaching the neutron drip line and in some nuclei they reach $50 \mathrm{MeV}$. However, they reduce substantially (down to maximum value of $\Delta E(Z, N) \approx 21$ $\mathrm{MeV}$ ) when PC-PK1 functional is removed from consideration. Two-neutron drip line of this functional is also located at substantially higher neutron numbers as compared with the ones obtained with other functionals. In addition, this functional is the major source of theoretical uncertainties in two-neutron separation energies. Parametric correlations leading to an over-parametrization of the isoscalar channel is a possible reason for such a unique behaviour of the CEDF PC-PK1. Theoretical uncertainties in two-neutron separation energies reveal clear importance of the uncertainties in the $N=184$ and $N=258$ spherical shell closures and in the location of the boundaries between the regions of prolate and oblate shapes.

- $\alpha$-decay properties, such as the $Q_{\alpha}$ values and the lifetimes $\tau_{\alpha}$, and related theoretical uncertainties have been investigated employing four empirical formulas and four CEDFs. While the predictive power of the models is relatively high on protonrich side of nuclear chart, it starts to deteriorate on approaching $N=184$. It is especially low in the nuclei around $Z \approx 108, N \approx 198$ [the region of the transition from prolate to oblate ground states] and in very neutron-rich nuclei located in the vicinity of two-proton drip line. However, the uncertainties in the latter region are not very important since the $\alpha$-decay in these nuclei is not expected to play any role in the r-process because of extremely large lifetimes.

- The distributions of the primary fission barriers in the $(Z, N)$ plane have been investigated with four employed CEDFs. Globally, the highest fission barriers are produced by the CEDF DD-ME2, and the lowest ones by the NL3* and PC-PK1 functionals. The results obtained with DD-PC1 are located between these two extremes but closer to the DDME2 ones. The presence of the band of nuclei with $N \approx 240$ in the $(Z, N)$ plane with low fission barriers, obtained in the calculations with the NL3* and PC-PK1 functionals, could have a drastic impact on the creation of superheavy elements in the r-process. The nuclear flow during most of neutron irradiation step of the r-process follows the neutron drip line and produces in tens of ms the heaviest drip line nuclei. However, this nuclear flow will most likely be terminated at $N \approx 240$ nuclei since fission will be much faster than neutron capture. On the contrary, the formation of superheavy elements in the r-process is more likely in the calculations based on the DD-ME2 and DD-PC1 functionals since the $(Z, N)$ region near neutron drip line is characterized by relatively high fission barriers and the band of nuclei with low fission barriers (similar to the one at $N \approx 240$ in the NL3* and PC-PK1 functionals) is absent.

- There are two major sources of theoretical uncertainties in the predictions of the heights of PFBs, namely, underlying single-particle structure mostly affecting the ground state properties and nuclear matter properties of employed CEDFs. For example, the increase of theoretical uncertainties for the ground states of the nuclei in the vicinity of the $N=184$ and $N=258$ spherical shell closures leads to an increase of theoretical uncertainties for their fission barriers. The functionals with nuclear matter properties located in the vicinity of empirical SET2b estimates [51] tend to produce higher fission barriers as compared with the predictions of the functionals the nuclear matter properties of which are located outside the limits of the SET2b constraint set. The problem of finding the best functional for the description of fission barriers is further complicated by the fact that the description of the ground state energies is to a degree decoupled from the description of fission barriers; the latter depends on the relative energies of the saddle and the ground state. As a consequence, good description of the ground state energies does not guarantee good description of the fission barriers and vice versa.

This is first ever systematic attempt within the covariant density functional theory to provide both the input for the r-process calculations which includes the ground state and fission properties of actinides and superheavy nuclei and the assessment of systematic theoretical uncertainties in the physical quantities of interest. As such it follows the ideology of all previous non-relativistic calcu- 
lations of relevance for the r-process of heavy and superheavy nuclei, which depend also on the fission processes, and assumes the axial symmetry of nuclei. This is a reasonable approximation for the ground state properties of the majority of nuclei; the only exception is transitional nuclei which are soft in $\gamma$-deformation. However, the restriction to axial symmetry leads to the fact that the calculated inner and outer fission barriers represent the upper limits and can be potentially lowered when the triaxiality is taken into account. The r-process simulations with the data obtained in this study will allow to limit the region of the $(Z, N)$ plane which has an impact on this process. The hope is that for this limited set of nuclei, systematic refined calculations taking into account the dynamical correlations and the triaxiality in the calculations of the part of the $\left(\beta_{2}, \gamma\right)$-plane covering ground state, inner fission barrier and second minimum as well as triaixiality and octupole deformation in the calculations of the part of the $\left(\beta_{2}, \beta_{3}, \gamma\right)$ plane covering second minimum, outer fission barrier and the region beyond that will be possible in the era of exascale computing.

Underlying single-particle structure and nuclear matter properties of CEDFs emerge as the major sources of theoretical uncertainties. However, they affect different physical observables in a different way. For example, theoretical uncertainties in the ground state quadrupole deformations are defined mostly by the uncertainties in the underlying single-particle structure. On the contrary, both factors contribute into theoretical uncertainties for fission barriers. The existence of appreciable theoretical uncertainties in the ground state and fission properties calls for a better covariant energy density functionals. The reduction of parametric correlations between the parameters of CEDFs is one possible way in that direction $[55,58]$. In addition, experimental studies of superheavy elements in the vicinity of the $Z=120$ and $N=184$ lines, planned at new facilities such as SHE factory [101], will hopefully provide critical data which will allow to discriminate the predictions of different models. Such information could be used for a better constraint of the CEDFs and thus to the reduction of substantial theoretical uncertainties in this region of nuclear chart which affect all physical observables of interest and have a direct impact on the modeling of the r-process.

\section{ACKNOWLEDGEMENT}

This material is based upon work supported by the US Department of Energy, National Nuclear Security Administration under Award No. DE-NA0002925, by the US Department of Energy, Office of Science, Office of Nuclear Physics under Award No. DE-SC0013037 and by Ghana Atomic Energy Commission, National Nuclear Research Institute, Ghana.

\section{Appendix A: Neglect of dynamical correlations in the fission barrier calculations}

Some of non-relativistic calculations, mentioned in the last paragraph of Sec. II, take into account dynamical correlations, but others not. Dynamical correlations are not taken into account in our calculations due to following reasons.

First, the analysis performed in Ref. [86] in the CDFTbased approach indicates that in most of the nuclei dynamical correlations modify fission barrier heights by less than $1 \mathrm{MeV}$ but increase substantially computational time. The only exceptions are the nuclei with soft potential energy surfaces the ground state energy minimum of which is located at spherical shape. Note that the absolute majority of the nuclei under consideration are deformed in the ground states (see Sect. III). Thus, the errors introduced into the fission barrier heights due to neglect of dynamical correlations are expected to be smaller than the ones which are coming from the selection of CEDFs (see Sect. V B below). In addition, theoretical uncertainties in fission barrier heights defined by their spreads (see Eq. (1)) are not expected to be modified much by the neglect of dynamical correlations. This is because in the majority of the cases the topology of potential energy surface of a given nucleus weakly depends on the underlying functional (see, for example, Figs. 7 and 8 in Ref. [47], Fig. 8 in Ref. [78] and supplemental material to Ref. [86]). As a consequence, dynamical correlations are expected to be comparable for different functionals and they will at least partially cancel each other in Eq. (1).

Second, the inner fission barriers are lowered when the triaxiality is taken into account (see Ref. [62] and references quoted therein) and the potential energy surfaces of the ground states in many superheavy nuclei are soft in $\gamma$-deformation (see Ref. [102] and Appendix B.) In the CDFT calculations, the outer fission barriers can also be affected by triaxial deformation ${ }^{11}$ via two mechanisms. In the first one, the saddle of reflection-symmetric triaxial fission path becomes lower in energy than the saddle of reflection-asymmetric (octupole-deformed) axial fission path due to underlying shell structure [78]. In the second mechanism, reflection-symmetric fission path and its saddle loose their axial symmetry and attain some degree of triaxiality [64, 69]. The investigations of the impact of dynamical correlations on fission barriers in triaxial calculations are very rare and quite limited in coverage. In non-relativistic frameworks, only limited set of actinides [104-107] and superheavy [108] nuclei have been studied so far. The impact of dynamical correlations on fission barriers of restricted set of superheavy nuclei along the

\footnotetext{
11 The important role of triaxiality in the description of outer fission barriers of actinides has also been discussed in the framework of microscopic+macroscopic approach in Ref. [103].
} 
$Z=120$ isotopic and $N=174,184$ isotonic chains has been studied in the CDFT-based framework in Ref. [86].

Third, dynamical calculations do not provide a unique answer because of underlying assumptions and approximations $[18,23,36,104,106,109,110]$. For example, there exist substantial differences between the predictions based on Adiabatic Time Dependent HFB (ATDHFB) and the Generator Coordinate method (GCM) [based on Gaussian Overlap Approximation (GOA)] schemes $[18,23,36,109]$. The differences between spontaneous fission half-lives $\tau_{S F}$ obtained in these two schemes could reach many orders of magnitude and increase with the decrease of the fissility-related parameter $Z^{2} / A$ [which is equivalent to the increase of neutron number within a given isotopic chain] (see Fig. 2 in Ref. [23]). Large differences between experimental and calculated $\tau_{S F}$ also exist; for example, in the $U$ isotopes these differences reach almost 20 orders of magnitudes when ATDHFB values for $\tau_{S F}$ are used [23]. As illustrated in Refs. $[104,106]$ on the example of the ${ }^{250,264} \mathrm{Fm}$ and ${ }^{240} \mathrm{Pu}$ nuclei, the inclusion of pairing fluctuations within a least action approach improves the agreement between the predicted $\tau_{S F}$ values and experiment. However, it remains to be seen whether that is a general conclusion applicable to all nuclei. In addition, such calculations are prohibitively expensive (in part, because of breaking of axial symmetry) and thus are not scalable to global calculations.

In addition, the treatment of the ground state energy $E_{0}$ (which is also tunneling energy for fission) relies on simplified approximations in the majority of the publications (see discussion in Ref. [86]). In microscopic calculations, tunneling energy is associated with the energy of collective ground state defined either in GCM [111] or in five-dimensional collective Hamiltonian (5DCH) [86]. The energy of collective ground state depends on softness (both in quadrupole deformation $\beta_{2}$ and in triaxial deformation $\gamma$ ) of collective energy surface in the vicinity of ground state minimum. It differs from approximate values substantially [86]; this could modify calculated $\tau_{S F}$ by several orders of magnitude $[86,108]$. These extremely large theoretical uncertainties in $\tau_{S F}$, coming from the selection of the method (ATDHFB versus GCM+GOA) and the treatment of the ground state energy, are the reasons why we have not attempted to calculate spontaneous fission half-lives in the present paper.

Fourth, there are some indications that the role of triaxiality can be reduced in dynamical calculations for some nuclei. For example, it was shown in Ref. [104] based on least-action calculations with Skyrme EDF $\mathrm{SkM}^{*}$ that pairing fluctuations act in the direction of restoration of axial symmetry along the fission path in the ${ }^{240} \mathrm{Pu}$ nucleus. This nucleus is characterized by relatively modest decrease (approximately $2 \mathrm{MeV}$ ) of inner fission barrier height by triaxiality in static calculations. Similar results have also been obtained for ${ }^{250,264} \mathrm{Fm}$ with similar formalism based on DD-PC1 CEDF in Ref. [106]. The calculations of Refs. [107, 108] based on least-action principle also indicate that the axial symmetry of fission pathway is restored in many nuclei; they are based on the DFT approach with Gogny D1S functional [107] and on macroscopic+microscopic method [108].

However, not in all nuclei the effect of triaxiality is eliminated by least-action principle. For example, the least-action fission pathway in ${ }^{264} \mathrm{Fm}$ is still characterized by triaxiality (although it is somewhat reduced by enhanced pairing as compared with static fission path) in the calculations based on Skyrme SkM* functional [104]. Note that in this nucleus the triaxiality has a large impact (slightly more than $4 \mathrm{MeV}$ ) on the height of inner fission barrier in static calculations. The calculations of Ref. [107] performed with Gogny D1S functionals also indicate that in some nuclei the least-action fission pathway goes through triaxial saddles. More systematic calculations $^{12}$ based on macroscopic + microscopic method show that the impact of the triaxiality on the least action fission pathway (and thus on spontaneous fission half-live) shows up in some nuclei with $Z=114$ and becomes much more pronounced in the $Z \geq 120$ nuclei [108]. Note that the tendency towards restoration of axial symmetry of the fission pathway in the least-action calculations may somewhat be underestimated in Refs. [107, 108] because of the neglect of pairing fluctuations.

The analysis of these publications suggests two possible situations in which the least-action fission path will most likely be characterized by triaxiality. In the first one, the decrease of the fission barrier by triaxiality in static calculations is substantial being on the order of $3-4 \mathrm{MeV}[104,108]$. In the second one, the ground state is oblate (or possibly soft in oblate-prolate direction [86]) so that the fission path across the $\gamma$-plane is shorter than the one along the $\gamma=0^{\circ}$ line [108]. As discussed in Sec. III and Appendix B only limited number of nuclei satisfy such conditions. Thus, the restriction to axial symmetry should be considered as a reasonable first approximation. However, one should keep in mind that the values obtained for fission barriers represent upper limits since their possible lowering due to triaxiality is neglected.

\section{Appendix B: Possible impact of triaxiality on inner fission barriers}

The restriction to axial symmetry is one of the approximations used in the present study which is a consequence of the global character of the study (see detailed discussion presented in the end of Sect. II and in Appendix A). In order to better understand for which nuclei this approximation may be violated (even in least-action calculations with pairing fluctuations included such as

\footnotetext{
12 These calculations are simplified as compared with quoted DFT calculations since they use fixed single-particle spectrum for all nuclei and neglect the deformations of higher order such as $\beta_{6}$ and $\beta_{8}$.
} 

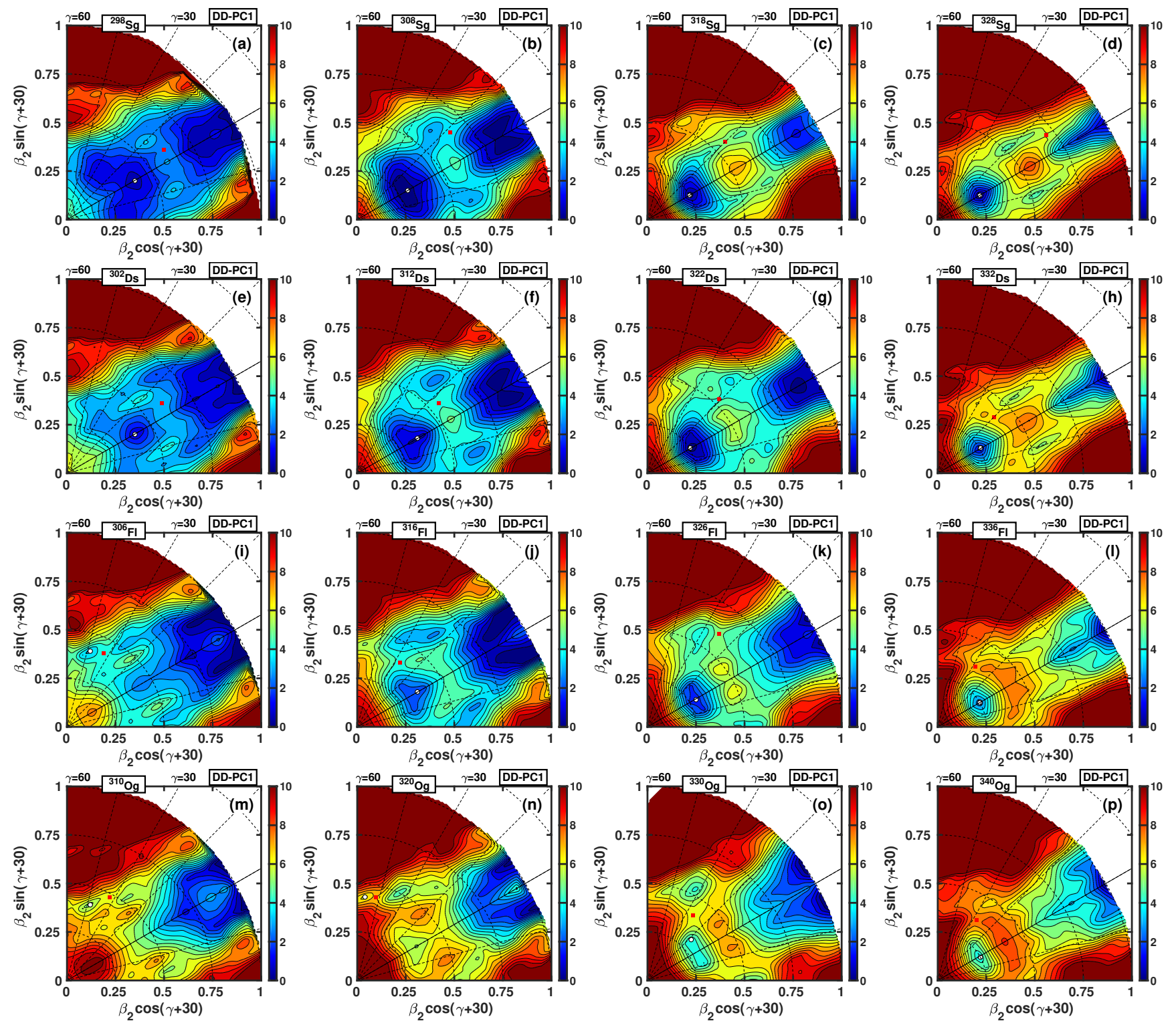

FIG. 18. Potential energy surfaces of the $\mathrm{Sg}(Z=106)$, Ds $(Z=110)$, Fl $(Z=114)$ and Og $(Z=118)$ isotopes with neutron numbers $N=192,202,212$ and 222 obtained in the triaxial RHB calculations with the DD-PC1 functional. Neutron number is increasing on going from left to right. The energy difference between two neighboring equipotential lines is equal to $0.5 \mathrm{MeV}$. The ground state minima and saddle points are shown by white circles and red solid squares, respectively.

those presented in Refs. $[104,106])$ we consider the examples of potential energy surfaces obtained in triaxial RHB calculations with the DD-PC1 functional. These PES calculated for the $\operatorname{Sg}(Z=106)$, Ds $(Z=110)$, $\mathrm{Fl}(Z=114)$ and $\mathrm{Og}(Z=118)$ isotopes with neutron numbers $N=192,202,212$ and 222 are presented in Fig. 18. They represent the extension of the calculations, executed in a more limited deformation space, the results of which are discussed in Sect. XI of Ref. [66]. The summary of the heights $E_{\text {triax }}^{B}$ of triaxial inner fission barriers and the decreases of the fission barrier heights due to triaxiality $\Delta E^{\text {gain }}$ are presented in Fig. 21 and Table II of this reference. Note that these superheavy nuclei are se- lected in such a way that they cover the part of nuclear chart characterized by both oblate and prolate ground states (see Fig. 5a).

The review of existing literature presented in Appendix A suggests two possible scenarios in which the leastaction fission path will most likely be characterized by triaxiality. In the first one, the decrease of the fission barrier by triaxiality in static calculations is substantial being on the order of $3-4 \mathrm{MeV}$ [104, 108]. Such decreases are observed in ${ }^{328} \mathrm{Sg}\left(\Delta E^{\text {gain }}=4.04 \mathrm{MeV}\right)$, ${ }^{310} \mathrm{Og}\left(\Delta E^{\text {gain }}=3.42 \mathrm{MeV}\right)$, and ${ }^{320} \mathrm{Og}\left(\Delta E^{\text {gain }}=4.93\right.$ $\mathrm{MeV}$ ) (see Figs. 18(d), (m) and (n) and Table II in Ref. $[66])$. In the second scenario, the ground state is oblate 
(or possibly soft in oblate-prolate direction [86]) so that the fission path across the $\gamma$-plane is shorter then the one along the $\gamma=0^{\circ}$ line [108]. This condition is satisfied only in the ${ }^{306} \mathrm{Fl}$ (see Fig. 18(i)) and ${ }^{310,320} \mathrm{Og}$ (see Fig. 18(m) and (n)) nuclei. Based on general features discussed in Refs. [104, 106], the analysis of PES of remaining nuclei (see Figs. 18 a, b, c, e, f, g, h, j, k, l, o and p) suggests that least-action fission pathway will be axial in these nuclei when pairing fluctuations are taken into account.

Whether one or another scenario takes place depends on the underlying shell structure (both at the ground state and saddle) defining the topology of potential energy surfaces in the $\left(\beta_{2}, \gamma\right)$ plane. Fig. 18 shows that the $N=192$ isotones are extremely soft in the $\gamma$-plane with clear tendency for the formation of near-oblate triaxial ground state minimum in the $\mathrm{Fl}$ and $\mathrm{Og}$ nuclei. However, static fission pathes from these minima are characterized by low fission barriers so these nuclei are expected to be unstable. Similar (but slightly less pronounced) situation is also seen for the $N=202$ isotones. The increase of neutron number to $N=212$ and 222 leads to a better localization of the ground state minimum at prolate shape and to an increase of fission barrier heights.
[1] Martínez-Pinedo, Selected topics in nuclear astrophysics, Eur. Phys. J. Spec. Topics 156, 123 (2008).

[2] V. Liccardo, M. Malheiro, M. S. Hussein, B. V. Carlson, and T. Frederico, Nuclear processes in astrophysics: recent progress, Eur. Phys. J. A54, 221 (2018).

[3] D. Kasen, B. Metzger, J. Barnes, E. Quataert, and E. Ramirez-Ruiz, Origin of the heavy elements in binary neutron-star mergers from a gravitational-wave event, Nature 551, 80 (2017).

[4] J. J. Cowan, C. Sneden, J. E. Lawler, A. Aprahamian, M. Wiescher, K. Langangke, G. Martínez-Pinedo, and F.-K. Thielemann, Making the heaviest elements in the universe: A review of the rapid neutron capture process, arkhiv: 1901.014110v1 [astro-ph.HE], subm. to Rev. Mod. Phys..

[5] F. K. Thielemann, M. Eichler, I. V. Panov, and B. Wehmeyer, Neutron star mergers and nucleosynthesis of heavy elements, Ann. Rev. Nucl. Part. Sci. 67, 253 (2017).

[6] B. P. Abbott et al., Gw170817: Observation of gravitational waves from a binary neutron star inspiral, Phys. Rev. Lett. 119, 161101 (2017).

[7] B. P. Abbott et al., Multi-messenger observations of a binary neutron star merger, Astrophys. J. Lett. 848, L12.

[8] O. Just, A. Bauswein, R. A. Pulpillo, S. Goriely, and H.T. Janka, Comprehensive nucleosynthesis analysis for ejecta of compact binary mergers, Mont. Not. R. Astr. Soc. 448, 541 (2015).

[9] Y. Sekiguchi, K. Kiuchi, K. Kyutoku, and M. Shibata, Dynamical mass ejection from binary neutron star mergers: Radiation-hydrodynamics study in general relativity, Phys. Rev. D 91, 064059 (2015).

[10] J. d. J. Mendoza-Temis, M.-R. Wu, K. Langanke, G. Martínez-Pinedo, A. Bauswein, and H.-T. Janka, Nuclear robustness of the $r$ process in neutron-star mergers, Phys. Rev. C 92, 055805 (2015).

[11] G. Martínez-Pinedo, D. Mocelj, N. Zinner, A. Kelić, K. Langanke, I. Panov, B. Pfeiffer, T. Rauscher, K.-H. Schmidt, and F.-K. Thielemann, The role of fission in the r-process, Prog. Part. Nucl. Phys. 59, 199 (2007).

[12] S. Goriely, The fundamental role of fission during r-process nucleosynthesis in neutron star mergers, Eur. Phys. J A51, 22 (2015).

[13] S. Goriely, J.-L. Sida, J.-F. Lemaître, S. Panebianco, N. Dubray, S. Hilaire, A. Bauswein, and H.-T. Janka,
New fission fragment distributions and $r$-process origin of the rare-earth elements, Phys. Rev. Lett. 111, 242502 (2013).

[14] M. Eichler, A. Arcones, A. Kelic, O. Korobkin, K. Langanke, T. Marketin, G. Martinez-Pinedo, I. Panov, T. Rauscher, S. Rosswog, C. Winteler, N. T. Zinner, and F.-K. Thielemann, The role of fission in neutron star mergers and its impact on the r-process peaks, Astrophys. J. 808, 30 (2015).

[15] I. Petermann, K. Langanke, G. Martínez-Pinedo, I. Panov, P.-G. Reinhard, and F.-K. Thielemann, Have superheavy elements been produced in nature?, Eur. Phys. J. A 48, 122 (2012).

[16] S. A. Giuliani, G. Martínez-Pinedo, L. M. Robledo, and M.-R. Wu, r-process calculations with a microsocpic description of the fission process, Acta Phys. Polonica B 48, 299 (2017).

[17] S. Goriely and M. Arnould, Waiting point approximation and canonical multi-event r-process revisited, Astr. and Astrophys. 312, 327 (1996).

[18] S. A. Giuliani, G. Martínez-Pinedo, and L. M. Robledo, Fission properties of superheavy nuclei for $r$-process calculations, Phys. Rev. C 97, 034323 (2018).

[19] A. Mamdouh, J. Pearson, M. Rayet, and F. Tondeur, Large-scale fission-barrier calculations with the etfsi method, Nucl. Phys. A 644, 389 (1998).

[20] S. Goriely, M. Samyn, and J. M. Pearson, Further explorations of skyrme-hartree-fock-bogoliubov mass formulas. vii. simultaneous fits to masses and fission barriers, Phys. Rev. C 75, 064312 (2007).

[21] J. Erler, K. Langanke, H. P. Loens, G. Martinez-Pinedo, and P.-G. Reinhard, Fission properties for $r$-process nuclei, Phys. Rev. C 85, 025802 (2012).

[22] P.-G. Reinhard, Nuclear density-functional theory and fission of super-heavy elements, Eur. Phys. J 312, 13 (2018).

[23] R. Rodríguez-Guzman, Y. M. Humadi, and L. M. Robledo, Microscopic description of fission in superheavy nuclei with the parametrization $\mathrm{d} 1 \mathrm{~m}^{*}$ of the gogny energy density functional, Eur. Phys. J. A56, 43 (2020).

[24] D. Vretenar, A. V. Afanasjev, G. A. Lalazissis, and P. Ring, Relativistic hartree-bogoliubov theory: Static and dynamic aspects of exotic nuclear structure, Phys. Rep. 409, 101 (2005).

[25] Extended density functionals in nuclear structure physics, Extended Density Functionals in Nuclear Struc- 
ture Physics, Lecture Notes in Physics, edited by G. A. Lalazissis, P. Ring, and D. Vretenar (Springer-Verlag, Heidelberg, 2004) Vol. 641 (2004).

[26] M. Bender, K. Rutz, P.-G. Reinhard, J. A. Maruhn, and W. Greiner, Shell structure of superheavy nuclei in selfconsistent mean-field models, Phys. Rev. C 60, 034304 (1999).

[27] E. V. Litvinova and A. V. Afanasjev, Dynamics of nuclear single-particle structure in covariant theory of particle-vibration coupling: From light to superheavy nuclei, Phys. Rev. C 84, 014305 (2011).

[28] W. Koepf and P. Ring, A relativistic description of rotating nuclei: the yrast line of ${ }^{20}$ ne, Nucl. Phys. A 493, 61 (1989).

[29] A. V. Afanasjev and H. Abusara, Time-odd mean fields in covariant density functional theory: nonrotating systems, Phys. Rev. C 81, 014309 (2010).

[30] U. Hofmann and P. Ring, A new method to calculate magnetic moments of relativistic mean field theories, Phys. Lett. B 214, 307 (1988).

[31] A. V. Afanasjev and P. Ring, Time-odd mean fields in the rotating frame: Microscopic nature of nuclear magnetism, Phys. Rev. C 62, 031302(R) (2000).

[32] A. V. Afanasjev and H. Abusara, Time-odd mean fields in covariant density functional theory: Rotating systems, Phys. Rev. C 82, 034329 (2010).

[33] J. Dobaczewski and J. Dudek, Time-odd components in the mean field of rotating superdeformed nuclei, Phys. Rev. C 52, 1827 (1995).

[34] N. Schunck, J. Dobaczewski, J. McDonnell, J. Moré, W. Nazarewicz, J. Sarich, and M. V. Stoitsov, Onequasiparticle states in the nuclear energy density functional theory, Phys. Rev. C 81, 024316 (2010).

[35] N. Hinohara, Z. P. Li, T. Nakatsukasa, T. Nikšić, and D. Vretenar, Effect of time-odd mean fields on inertial parameters of the quadrupole collective hamiltonian, Phys. Rev. C 85, 024323 (2012).

[36] S. A. Giuliani and L. M. Robledo, Non-perturbative collective inertias for fission: A comparative study, Phys. Lett. B 787, 134 (2018).

[37] P. G. Reinhard and W. Nazarewicz, Information content of a new observable: The case of the nuclear neutron skin, Phys. Rev. C 81, 051303(R) (2010).

[38] J. Dobaczewski, W. Nazarewicz, and P.-G. Reinhard, Error estimates of theoretical models: a guide, J. Phys. G 41, 074001 (2014).

[39] S. E. Agbemava, A. V. Afanasjev, D. Ray, and P. Ring, Global performance of covariant energy density functionals: Ground state observables of even-even nuclei and the estimate of theoretical uncertainties, Phys. Rev. C 89, 054320 (2014).

[40] M. Bender, P.-H. Heenen, and P.-G. Reinhard, Selfconsistent mean-field models for nuclear structure, Rev. Mod. Phys. 75, 121 (2003).

[41] J. Boguta and R. Bodmer, Nucl. Phys. A292, 413 (1977).

[42] G. A. Lalazissis, T. Nikšić, D. Vretenar, and P. Ring, New relativistic mean-field interaction with densitydependent meson-nucleon couplings, Phys. Rev. C 71, 024312 (2005).

[43] G. A. Lalazissis, S. Karatzikos, R. Fossion, D. P. Arteaga, A. V. Afanasjev, and P. Ring, The effective force nl3 revisited, Phys. Lett. B671, 36 (2009).

[44] T. Nikšić, D. Vretenar, and P. Ring, Relativistic nu- clear energy density functionals: adjusting parameters to binding energies, Phys. Rev. C 78, 034318 (2008).

[45] S. Typel and H. H. Wolter, Nucl. Phys. A656, 331 (1999).

[46] S. E. Agbemava, A. V. Afanasjev, and A. Taninah, Propagation of statistical uncertainties in covariant density functional theory: Ground state observables and single-particle properties, Phys. Rev. C 99, 014318 (2019).

[47] S. E. Agbemava, A. V. Afanasjev, D. Ray, and P. Ring, Assessing theoretical uncertainties in fission barriers of superheavy nuclei, Phys. Rev. C 95, 054324 (2017).

[48] S. E. Agbemava, A. V. Afanasjev, and P. Ring, Octupole deformation in the ground states of even-even nuclei: a global analysis within the covariant density functional theory, Phys. Rev. C 93, 044304 (2016).

[49] A. V. Afanasjev and S. E. Agbemava, Covariant energy density functionals: Nuclear matter constraints and global ground state properties, Phys. Rev. C 93, 054310 (2016).

[50] P. W. Zhao, Z. P. Li, J. M. Yao, and J. Meng, New parametrization for the nuclear covariant energy density functional with a point-coupling interaction, Phys. Rev. C 82, 054319 (2010).

[51] M. Dutra, O. Lourenco, S. S. Avancini, B. V. Carlson, A. Delfino, D. P. Menezes, C. Providencia, S. Typel, and J. R. Stone, Relativistic mean-field hadronic models under nuclear matter constraints, Phys. Rev. C 90, 055203 (2014).

[52] P.-G. Reinhard, M. Rufa, J. Maruhn, W. Greiner, and J. Friedrich, Nuclear ground state properties in a relativistic meson field model, Z. Phys. A 323, 13 (1986).

[53] R. Furnstahl, B. D. Serot, and H.-B. Tang, A chiral effective lagrangian for nuclei, Nucl. Phys. A 615, 441 (1997).

[54] X. Roca-Maza, X. Viñas, M. Centelles, P. Ring, and P. Schuck, Relativistic mean-field interaction with density-dependent meson-nucleon vertices based on microscopical calculations, Phys. Rev. C 84, 054309 (2011).

[55] T. Nikšić, M. Imbrišak, and D. Vretenar, "sloppy" nuclear energy density functionals. ii. finite nuclei, Phys. Rev. C 95, 054304 (2017).

[56] B. Kumar, S. Singh, B. Agrawal, and S. Patra, New parameterization of the effective field theory motivated relativistic mean field model, Nucl. Phys. A 966, 197 (2017).

[57] E. Yüksel, T. Marketin, and N. Paar, Optimizing the relativistic energy density functional with nuclear ground state and collective excitation properties, Phys. Rev. C 99, 034318 (2019).

[58] A. Taninah, S. E. Agbemava, A. V. Afanasjev, and P. Ring, Parametric correlations in energy density functionals, Phys. Lett. B 800, 135065 (2020).

[59] S. E. Agbemava, A. V. Afanasjev, T. Nakatsukasa, and P. Ring, Covariant density functional theory: Reexamining the structure of superheavy nuclei, Phys. Rev. C 92, 054310 (2015).

[60] K. Q. Lu, Z. X. Li, Z. P. Li, J. M. Yao, and J. Meng, Global study of beyond-mean-field correlation energies in covariant energy density functional theory using a collective hamiltonian method, Phys. Rev. C 91, 027304 (2015).

[61] S. E. Agbemava and A. V. Afanasjev, Octupole defor- 
mation in the ground states of even-even $z \sim 96, n \sim$ 196 actinides and superheavy nuclei, Phys. Rev. C 96, 024301 (2017).

[62] H. Abusara, A. V. Afanasjev, and P. Ring, Fission barriers in actinides in covariant density functional theory: the role of triaxiality, Phys. Rev. C 82, 044303 (2010).

[63] V. Prassa, T. Nikšić, G. A. Lalazissis, and D. Vretenar, Relativistic energy density functional description of shape transitions in superheavy nuclei, Phys. Rev. C 86, 024317 (2012).

[64] B.-N. Lu, J. Zhao, E.-G. Zhao, and S.-G. Zhou, Multidimensionally-constrained relativistic mean-field models and potential-energy surfaces of actinide nuclei, Phys. Rev. C 89, 014323 (2014).

[65] A. V. Afanasjev, S. E. Agbemava, and A. Gyawali, Hyperheavy nuclei: Existence and stability, Phys. Lett. B 782, 533 (2018).

[66] S. E. Agbemava, A. V. Afanasjev, A. Taninah, and A. Gyawali, Extension of the nuclear landscape to hyperheavy nuclei, Phys. Rev. C 99, 034316 (2019).

[67] A. V. Afanasjev, S. E. Agbemava, and A. Taninah, Exploring nuclear exotica at the limits, Acta Phys. Polonica B 13, 347 (2020).

[68] Q. S. Zhang, Z. M. Niu, Z. P. Li, J. M. Yao, and J. Meng, Global dynamical correlation energies in covariant density functional theory: cranking approximation, Frontiers of Physics 9, 529 (2014).

[69] B.-N. Lu, E.-G. Zhao, and S.-G. Zhou, Potential energy surfaces of actinide nuclei from a multidimensional constrained covariant density functional theory: Barrier heights and saddle point shapes, Phys. Rev. C 85, 011301 (2012).

[70] A. V. Afanasjev, P. Ring, and J. König, Cranked relativistic hartree-bogoliubov theory: formalism and application to the superdeformed bands in the $a \sim 190$ region., Nucl. Phys. A676, 196 (2000).

[71] H. Kucharek and P. Ring, Relativistic field theory of superfluidity in nuclei, Z. Phys. A 339, 23 (1991).

[72] P. Ring, Relativistic mean field theory in finite nuclei, Prog. Part. Nucl. Phys. 37, 193 (1996).

[73] S. Karatzikos, A. V. Afanasjev, G. A. Lalazissis, and P. Ring, The fission barriers in actinides and superheavy nuclei in covariant density functional theory, Phys. Lett. B 689, 72 (2010).

[74] Y. Tian, Z. Y. Ma, and P. Ring, A finite range pairing force for density functional theory in superfluid nuclei, Phys. Lett. B 676, 44 (2009).

[75] J. F. Berger, M. Girod, and D. Gogny, Comp. Phys. Comm. 63, 365 (1991).

[76] A. V. Afanasjev and O. Abdurazakov, Pairing and rotational properties of actinides and superheavy nuclei in covariant density functional theory, Phys. Rev. C 88, 014320 (2013).

[77] J. Dobaczewski, A. V. Afanasjev, M. Bender, L. M. Robledo, and Y. Shi, Properties of nuclei in the nobelium region studied within the covariant, skyrme, and gogny energy density functionals, Nucl. Phys. A 944, 388 (2015).

[78] H. Abusara, A. V. Afanasjev, and P. Ring, Fission barriers in covariant density functional theory: extrapolation to superheavy nuclei., Phys. Rev. C 85, 024314 (2012).

[79] P. Ring and P. Schuck, The Nuclear Many-Body Problem (Springer-Verlag, Berlin) (1980).

[80] P. Bonche, H. Flocard, and P. H. Heenen, Solution of the skyrme hf+bcs equation on a $3 \mathrm{~d}$ mesh, Comp. Phys. Comm. 171, 49 (2005).

[81] T. Bürvenich, M. Bender, J. A. Maruhn, and P.-G. Reinhard, Systematics of fission barriers in superheavy elements, Phys. Rev. C 69, 014307 (2004).

[82] A. Staszczak, A. Baran, J. Dobaczewski, and W. Nazarewicz, Microscopic description of complex nuclear decay: Multimodal fission, Phys. Rev. C 80, 014309 (2009).

[83] P. Möller, A. J. Sierk, T. Ichikawa, A. Iwamoto, R. Bengtsson, H. Uhrenholt, and S. Åberg, Heavyelement fission barriers, Phys. Rev. C 79, 064304 (2009).

[84] W. D. Myers and W. J. Świațecki, Thomas-fermi fission barriers, Phys. Rev. C 60, 014606 (1999).

[85] M. Arnould and S. Goriely, Astronuclear physics: a tale of the atomic nuclei in the skies, arXiv:2001.11228v1 [astro-ph.SR (2020).

[86] Z. Shi, A. V. Afanasjev, Z. P. Li, and J. Meng, Superheavy nuclei in a microscopic collective hamiltonian approach: The impact of beyond-mean-field correlations on ground state and fission properties, Phys. Rev. C 99, 064316 (2019).

[87] T. Sil, S. K. Patra, B. K. Sharma, M. Centelles, and X. Viñas, Superheavy nuclei in a relativistic effective lagrangian model, Phys. Rev. C 69, 044315 (2004).

[88] M. Bender, W. Nazarewicz, and P.-G. Reinhard, Shell stabilization of super- and hyperheavy nuclei without magic gaps, Phys. Lett. B 515, 42 (2001).

[89] W. Zhang, J. Meng, S. Zhang, L. Geng, and H. Toki, Magic numbers for superheavy nuclei in relativistic continuum hartree-bogoliubov theory, Nucl. Phys. A 753, 106 (2005).

[90] A. V. Afanasjev, S. E. Agbemava, D. Ray, and P. Ring, Neutron drip line: Single-particle degrees of freedom and pairing properties as sources of theoretical uncertainties, Phys. Rev. C 91, 014324 (2015).

[91] Evaluated Nuclear Structure Data File (ENSDF) located at the website (http://www.nndc.bnl.gov/ensdf/) of Brookhaven National Laboratory. ENSDF is based on the publications presented in Nuclear Data Sheets (NDS) which is a standard for evaluated nuclear data. (2015).

[92] X. Xia, Y. Lim, P. Zhao, H. Liang, X. Qu, Y. Chen, H. Liu, L. Zhang, S. Zhang, Y. Kim, and J. Meng, The limits of the nuclear landscape explored by the relativistic continuum hartree-bogoliubov theory, At. Data Nucl. Data Tables 121-122, 1 (2018).

[93] A. V. Afanasjev, S. E. Agbemava, D. Ray, and P. Ring, Nuclear landscape in covariant density functional theory, Phys. Lett. B 726, 680 (2013).

[94] M. Wang, G. Audi, A. H. Wapstra, F. G. Kondev, M. MacCormick, X. Xu, and B. Pfeiffer, Chinese Physics C36, 1603 (2012).

[95] J. V. E. Viola and G. T. Seaborg, J. Inorg. Nucl. Chem. 28, 741 (1966).

[96] A. Sobiczewski, Z. Patyk, and S. Cwiok, Deformed superheavy nuclei, Physics Letters B 224, 1 (1989).

[97] T. Dong and Z. Ren, New calculations of $\hat{\mathrm{I}} \pm$-decay halflives by the viola-seaborg formula., Eur. Phys. J. A26, 69 (2005).

[98] A. Budaca, R. Budaca, and I. Silisteanu, Extended systematics of alpha decay half lives for exotic superheavy nuclei, Nucl. Phys. A 951, 60 (2016). 
[99] G. Royer, Alpha emission and spontaneous fission through quasi-molecular shapes, J. Phys. G 26, 1149 (2000).

[100] C. Y. Wong, Toroidal and spherical bubble nuclei, Annals of Physics 77, 279 (1973).

[101] Y. T. Oganessian and S. N. Dmitriev, Synthesis and study of properties of superheavy atoms. factory of superheavy elements, Rus. Chem. Rev. 85, 901 (2016).

[102] S. Ćwiok, P.-H. Heenen, and W. Nazarewicz, Shape coexitence and triaxiality in the superheavy nuclei, Nature 433, 705 (2005).

[103] A. Dobrowolski, K. Pomorski, and J. Bartel, Fission barriers in a macroscopic-microscopic model, Phys. Rev. C 75, 024613 (2007).

[104] J. Sadhukhan, J. Dobaczewski, W. Nazarewicz, J. A. Sheikh, and A. Baran, Pairing-induced speedup of nuclear spontaneous fission, Phys. Rev. C 90, 061304(R) (2014).

[105] K. Benrabia, D. E. Medjadi, M. Imadalou, and P. Quentin, Triaxial quadrupole dynamics and the inner fission barrier of some heavy even-even nuclei, Phys. Rev. C 96, 034320 (2017).

[106] J. Zhao, B.-N. Lu, T. Nikšić, D. Vretenar, and S.-
G. Zhou, Multidimensionally-constrained relativistic mean-field study of spontaneous fission: Coupling between shape and pairing degrees of freedom, Phys. Rev. C 93, 044315 (2016).

[107] J.-P. Delaroche, M. Girod, H. Goutte, and J. Libert, Structure properties of even-even actinides at normal and superdeformed shapes analysed using the gogny force, Nucl. Phys. A 771, 103 (2006).

[108] R. Gherghescu, J. Skalski, Z. Patyk, and A. Sobiczewski, Non-axial shapes in spontaneous fission of superheavy nuclei, Nucl. Phys. A 651, 237 (1999).

[109] N. Schunck and L. M. Robledo, Microscopic theory of nuclear fission: A review, Rep. Prog. Phys. 79, 116301 (2016).

[110] S. A. Giuliani, Z. Matheson, W. Nazarewicz, E. Olsen, P.-G. Reinhard, J. Sadhukhan, B. Schuetrumpf, N. Schunck, and P. Schwerdtfeger, Colloquium: Superheavy elements: Oganesson and beyond, Rev. Mod. Phys. 91, 011001 (2019).

[111] N. Schindzielorz, J. Erler, P. Klupfel, P. G. Reinhard, and G. Hager, Int. J. Mod. Phys. E 18, 773 (1981). 